\title{
Single-nucleus transcriptomic landscape of primate hippocampal aging
}

\author{
Hui Zhang ${ }^{1,6}$, Jiaming $\mathrm{Li}^{4,6,7,10,11}$, Jie Ren ${ }^{4,5,6,7,10} \mathbb{( 1 )}$, Shuhui Sun ${ }^{1,5,13}$, Shuai Ma ${ }^{1,5,13}$, Weiqi Zhang ${ }^{4,5,6,7,10}{ }_{\mathbb{1}}$, \\ Yang $\mathrm{Yu}^{12,14}$, Yusheng Cai ${ }^{1,5,13}$, Kaowen Yan ${ }^{1,5,13}$, Wei $\mathrm{Li}^{2,5,6,13}$, Baoyang $\mathrm{Hu}^{2,5,6,13}$, Piu Chan ${ }^{3}$, \\ Guo-Guang Zhao ${ }^{3,9}$, Juan Carlos Izpisua Belmonte ${ }^{15}$, Qi Zhou ${ }^{2,5,6,13}$, Jing Qu ${ }^{2,5,6,13 \bowtie}{ }_{\mathbb{D}}$, Si Wang $^{3,8}$ (D), \\ Guang-Hui Liu ${ }^{1,3,5,6,13 \bowtie}$
}

${ }^{1}$ State Key Laboratory of Membrane Biology, Institute of Zoology, Chinese Academy of Sciences, Beijing 100101, China

${ }^{2}$ State Key Laboratory of Stem Cell and Reproductive Biology, Institute of Zoology, Chinese Academy of Sciences, Beijing 100101, China

${ }^{3}$ Advanced Innovation Center for Human Brain Protection, National Clinical Research Center for Geriatric Disorders, Xuanwu Hospital Capital Medical University, Beijing 100053, China

${ }^{4}$ CAS Key Laboratory of Genomic and Precision Medicine, Beijing Institute of Genomics, Chinese Academy of Sciences, Beijing 100101, China

${ }^{5}$ Institute for Stem Cell and Regeneration, Chinese Academy of Sciences, Beijing 100101, China

${ }^{6}$ University of Chinese Academy of Sciences, Beijing 100049, China

${ }^{7}$ China National Center for Bioinformation, Beijing 100101, China

${ }^{8}$ Aging Translational Medicine Center, Xuanwu Hospital, Capital Medical University, Beijing 100053, China

${ }^{9}$ Department of Neurosurgery, Xuanwu Hospital, Capital Medical University, Beijing 100053, China

${ }^{10}$ Sino-Danish College, University of Chinese Academy of Sciences, Beijing 101408, China

11 Sino-Danish Center for Education and Research, Beijing 101408, China

12 Department of Obstetrics and Gynecology, Center for Reproductive Medicine, Peking University Third Hospital, Beijing 100191, China

${ }^{13}$ Beijing Institute for Stem Cell and Regenerative Medicine, Beijing 100101, China

${ }^{14}$ Stem Cell Research Center, Peking University Third Hospital, Beijing 100191, China

15 Gene Expression Laboratory, Salk Institute for Biological Studies, La Jolla, CA, USA

$\square$ Correspondence: qujing@ioz.ac.cn (J. Qu), wangsi@xwh.ccmu.edu.cn (S. Wang), ghliu@ioz.ac.cn (G.-H. Liu)

Received April 11, 2021 Accepted April 24, 2021

\begin{abstract}
The hippocampus plays a crucial role in learning and memory, and its progressive deterioration with age is functionally linked to a variety of human neurodegenerative diseases. Yet a systematic profiling of the aging effects on various hippocampal cell types in primates is still missing. Here, we reported a variety of new aging-associated phenotypic changes of the primate hippocampus. These include, in particular, increased DNA damage and heterochromatin erosion with time, alongside loss of proteostasis and elevated inflammation. To understand their cellular and
\end{abstract}

Hui Zhang, Jiaming Li and Jie Ren have contributed equally.

Supplementary Information The online version contains supplementary material available at https://doi.org/10.1007/s13238-02100852-9. molecular causes, we established the first single-nucleus transcriptomic atlas of primate hippocampal aging. Among the 12 identified cell types, neural transiently amplifying progenitor cell (TAPC) and microglia were most affected by aging. In-depth dissection of gene-expression dynamics revealed impaired TAPC division and compromised neuronal function along the neurogenesis trajectory; additionally elevated pro-inflammatory responses in the aged microglia and oligodendrocyte, as well as dysregulated coagulation pathways in the aged endothelial cells may contribute to a hostile microenvironment for neurogenesis. This rich resource for understanding primate hippocampal aging may provide potential diagnostic biomarkers and therapeutic interventions against age-related neurodegenerative diseases.

KEYWORDS aging, hippocampus, primate, single-cell RNA sequencing 
A

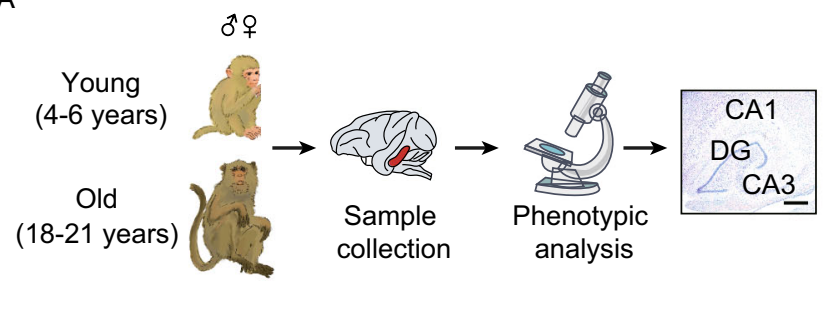

B Nissl

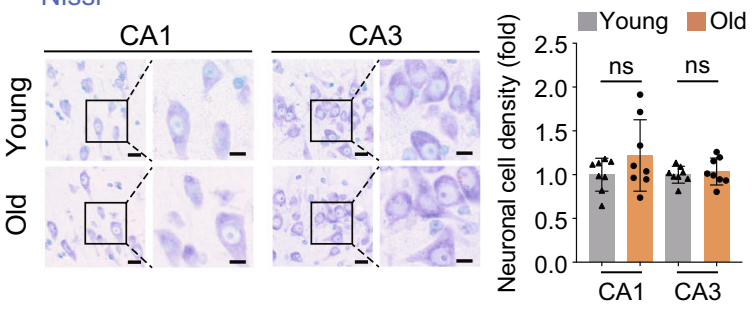

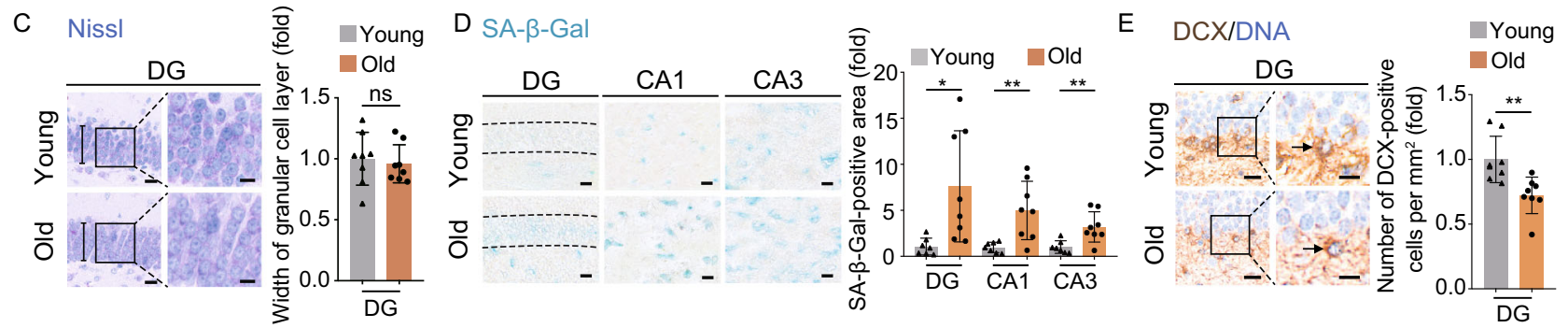

F Aggresome/DNA
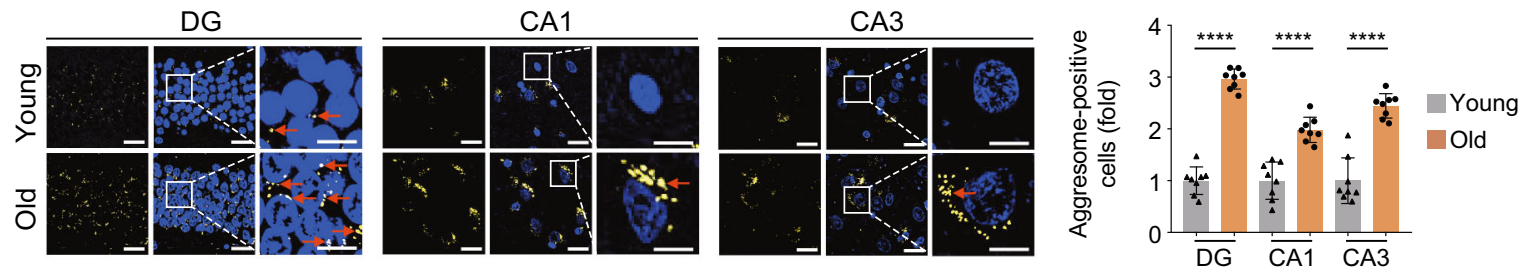

G
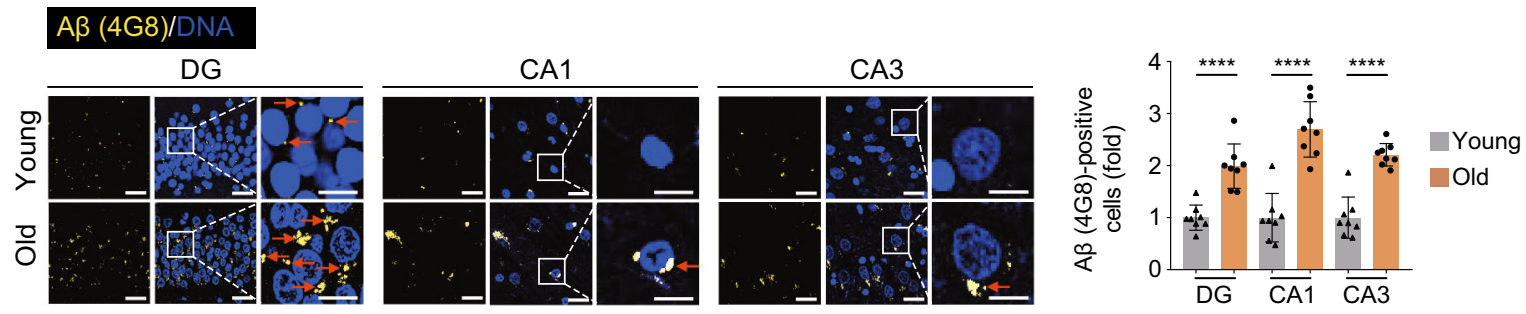

H A (1-40)/DNA
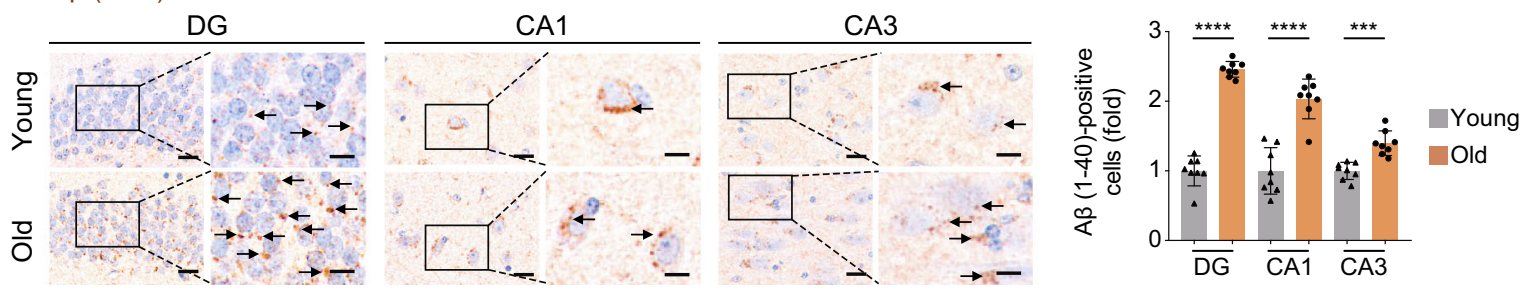
Figure 1. Aging-related phenotypes of the cynomolgus monkey hippocampus. (A) Flow chart of the phenotypic analysis on the hippocampal tissues collected form young and old monkeys. Representative image of Nissl staining in the hippocampus is shown (right panel, dentate gyrus (DG), CA1 region (CA1) and CA3 region (CA3), scale bar, $700 \mu \mathrm{m})$. (B) NissI staining in $\mathrm{CA} 1$ and $\mathrm{CA} 3$ regions of the hippocampus from young and old monkeys. Representative images are shown on the left; neuronal cell densities in corresponding regions are quantified as fold changes (old vs. young), shown as means \pm SEM on the right. Scale bars, $20 \mu \mathrm{m}$ and $10 \mu \mathrm{m}$ (zoomed-in image). Young, $n=8$; old, $n=8$ monkeys. ns, not significant. (C) Nissl staining in the dentate gyrus (DG) from young and old monkeys. Representative images are shown on the left; the widths of the granular cell layers in the DG are quantified as fold changes (old vs. young), shown as means \pm SEM on the right. Scale bars, $20 \mu \mathrm{m}$ and $10 \mu \mathrm{m}$ (zoomed-in image). Young, $n=8$; old, $n=8$ monkeys. $\mathrm{ns}$, not significant. (D) SA- $\beta$-Gal staining in the indicated regions of the hippocampus from young and old monkeys. Representative images are shown on the left; SA- $\beta$-Gal positive areas in the DG, CA1 and CA3 regions are quantified as fold changes (young vs. old), shown as means \pm SEM on the right. Scale bar, $20 \mu \mathrm{m}$. Young, $n=7$; old, $n=8$ monkeys. ${ }^{\star} P<0.05$; ${ }^{* \star} P<0.01$. (E) Immunohistochemical staining of DCX in the DG region of the hippocampus from young and old monkeys. Representative images are shown on the left; DCX-positive cells are quantified as fold changes of their numbers in the old DG vs. in young counterparts, shown as means \pm SEM on the right. Black arrows indicate the DCX-positive cells. Scale bars, $20 \mu \mathrm{m}$ and $10 \mu \mathrm{m}$ (zoomed-in image). Young, $n=8$; old, $n=8$ monkeys. ${ }^{* *} P<0.01$. (F) Aggresome staining in the indicated regions of the hippocampus from young and old monkeys. Representative images are shown on the left; aggresome-positive cells are quantified as fold changes of their numbers in the old DG, CA1 and CA3 regions vs. in young counterparts, shown as means \pm SEM on the right. Red arrows indicate the aggresome-positive cells. Scale bars, $20 \mu \mathrm{m}$ and $10 \mu \mathrm{m}$ (zoomed-in image). Young, $n=8$; old, $n=8$ monkeys. ${ }^{* * * *} P<0.0001$. (G) Immunofluorescence staining of $A \beta$ (4G8) accumulation in the indicated regions of the hippocampus from young and old monkeys. Representative images are shown on the left; $A \beta$ (4G8)-positive cells are quantified as fold changes of their numbers in the old DG, CA1 and $\mathrm{CA} 3$ regions vs. young counterparts, shown as means \pm SEM on the right. Red arrows indicate the $A \beta$ (4G8)-positive cells. Scale bars, $20 \mu \mathrm{m}$ and $10 \mu \mathrm{m}$ (zoomed-in image). Young, $n=8$; old, $n=8$ monkeys. ${ }^{* * * *} P<0.0001$. $(\mathrm{H})$ Immunofluorescence staining of $A \beta(1-40)$ accumulation in the indicated regions of the hippocampus from young and old monkeys. Representative images are shown on the left; quantitative data for the relative $A \beta$ (1-40)-positive cells in the DG, CA1 and CA3 regions are shown as means \pm SEM on the right. The relative fold of number of $A \beta$ (1-40)-positive cells was obtained by normalizing the number of $A \beta$ (1-40)-positive cells of the old monkey with the young monkey. Black arrows indicate the $A \beta(1-40)$-positive cell. Scale bars, $20 \mu \mathrm{m}$ and $10 \mu \mathrm{m}$ (zoomed-in image). Young, $n=8$; old, $n=8$ monkeys. ${ }^{* *} P<0.001,{ }^{* * *} P<0.0001$.

\section{INTRODUCTION}

Aging is a major risk factor for human neurodegenerative disorders, such as Alzheimer's disease (AD), which is rapidly rising in prevalence with age and has become the most pressing challenge to be tackled. The hippocampus plays a crucial role in spatial and episodic learning and memory (Morrison and Baxter, 2012; Fan et al., 2017; Kuhn et al., 2018). Age-related decline in hippocampal functions manifests as a profound yet inevitable impairment in cognitive abilities and increases vulnerability to AD (Morrison and Baxter, 2012; Fan et al., 2017; Hou et al., 2019). Therefore, a comprehensive understanding of the underlying mechanisms of hippocampal aging is of scientific and clinical importance.

Although still being debated, adult neurogenesis continues in the subgranular zone (SGZ) of the dentate gyrus (DG) within the mammalian hippocampus (Boldrini et al., 2018), critical to contextual learning and episodic memory (Kuhn et al., 2018; Navarro Negredo et al., 2020). In the adult DG, radial glia-like quiescent neural stem cell (NSC) allows for the addition of newborn neuron throughout life, initiating the neurogenesis trajectory through the transition into activation, proliferation, and then generation of transiently amplifying progenitor cell (TAPC) (Kempermann et al., 2015; Li et al., 2015; Navarro Negredo et al., 2020). TAPC then gives rise to neuroblast, which further differentiates into immature and then mature granule neuron or glial cell (Aimone et al., 2014; Encinas et al., 2011; Navarro Negredo et al., 2020). Both NSC and their progenies interact extensively with several kinds of neurogenic niche cells, including oligodendrocyte, microglia, and cells comprising the neurovasculature (Aimone et al., 2014; Fan et al., 2017). Although a decline in the volume of the aged anterior hippocampus was observed in some studies (Malykhin et al., 2008), others reported conflicting findings or altered hippocampal shape rather than its volume during the aging process (Head et al., 2005; Yang et al., 2013). Through histological analysis, compromised neurogenesis, a gradual loss of synaptic plasticity, elevated microglia activation, and diminished angiogenesis have been identified as major age-associated changes in the hippocampus, which may result in age-dependent cognitive decline (Leuner et al., 2007; Morrison and Baxter, 2012; Fan et al., 2017; Navarro Negredo et al., 2020). Although extensive studies have been reported on the morphological characteristics of the hippocampus during aging, the complexity of underlying cellular and molecular alterations has never been revealed comprehensively and at single-cell resolution.

Due to ethical restrictions, it is difficult to obtain diseasefree human brain tissues, including the hippocampus from both young and old individuals. Therefore, unbiased studies of human hippocampal degeneration are not feasible. Since non-human primates (NHPs) share similar genetic, physiological, and neurological characteristics with humans (Chen et al., 2012, 2016; Chen et al., 2017; Colman, 2018; Zhang 
A $y H 2 A . X / D N A$
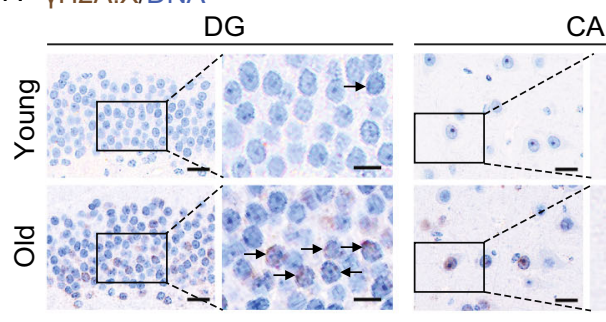

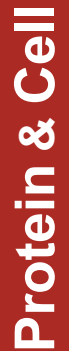

B

dsDNA/DNA

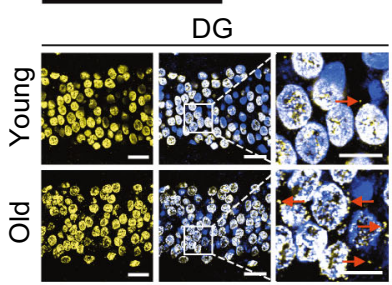

C H3K9me3/DNA

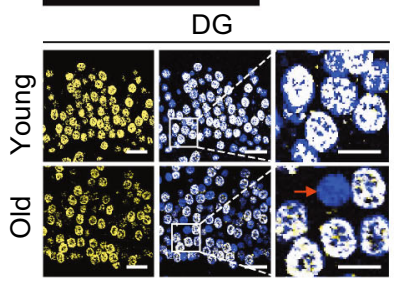

D HP1Y/DNA

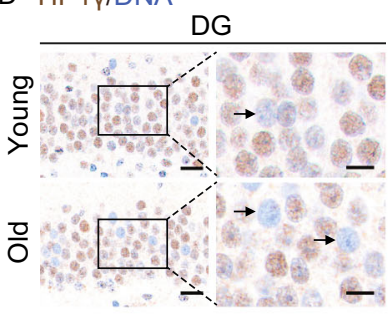

\section{E LINE-1 ORF2-p/DNA}

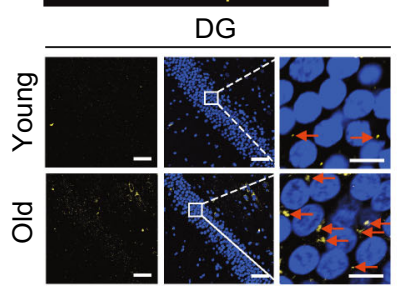

CA1
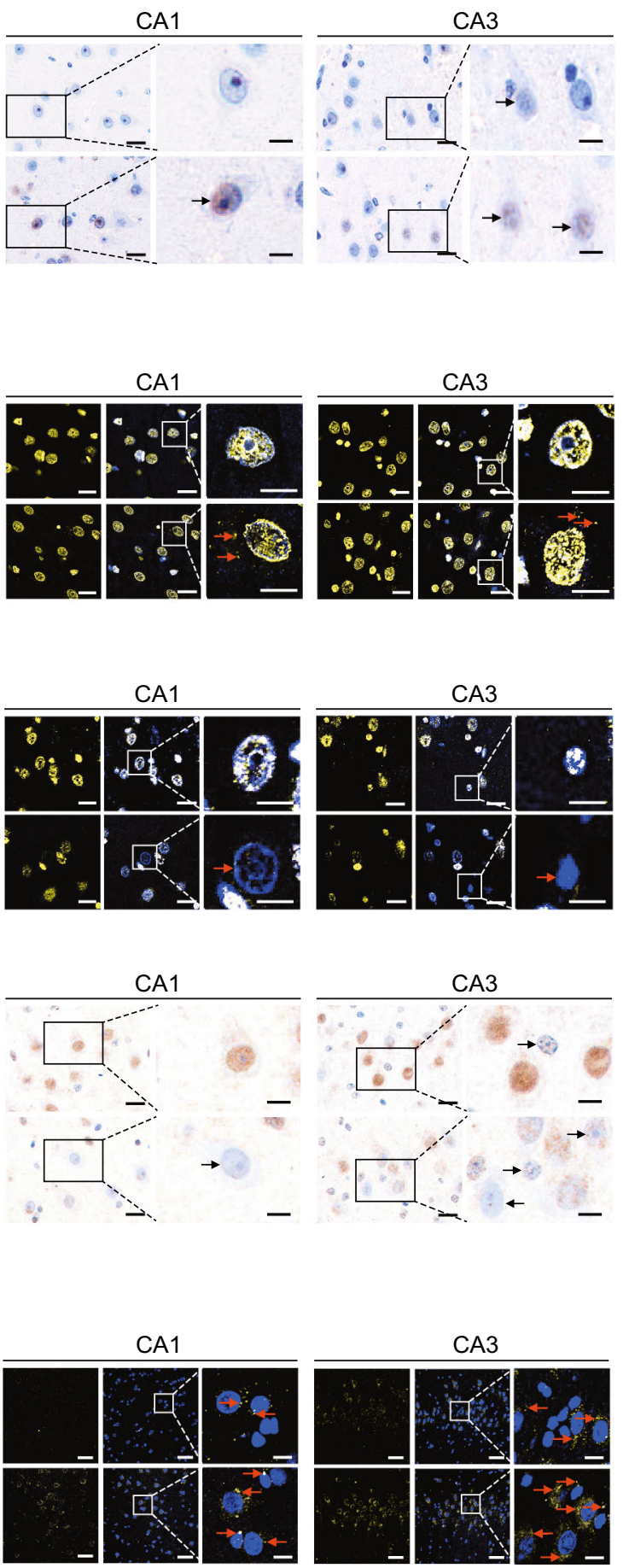
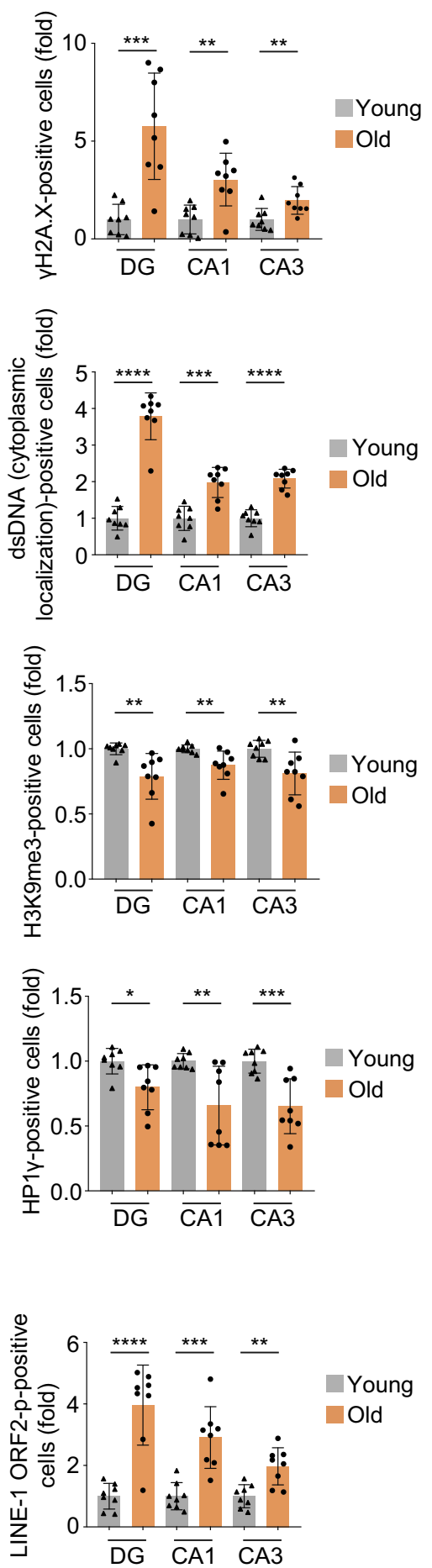
Figure 2. Aging-related loss of genomic and epigenomic stability in the monkey hippocampus. (A) Immunohistochemical staining of $Y H 2 A . X$ in the indicated regions of the hippocampus from young and old monkeys. Representative images are shown on the left; $\mathrm{YH}$ 2A.X-positive cells are quantified as fold changes of their numbers in the old DG, CA1 and CA3 regions vs. in young counterparts, shown as means \pm SEM on the right. Black arrows indicate the $\mathrm{YH} 2 \mathrm{~A} . \mathrm{X}$ positive cells. Scale bars, $20 \mu \mathrm{m}$ and $10 \mu \mathrm{m}$ (zoomed-in image). Young, $n=8$; old, $n=8$ monkeys. ${ }^{* * *} P<0.001$; ${ }^{* *} P<0.01$. (B) Immunofluorescence staining of dsDNA in the indicated regions of the hippocampus from young and old monkeys. Representative images are shown on the left; cytoplasmic dsDNA-positive cells are quantified as fold changes of their numbers in the old DG, CA1 and CA3 regions vs. in young counterparts, shown as means \pm SEM on the right. Red arrows indicate the cytoplasm-localized dsDNA. Scale bars, $20 \mu \mathrm{m}$ and $10 \mu \mathrm{m}$ (zoomed-in image). Young, $n=8$; old, $n=8$ monkeys. ${ }^{\star * \star \star} P<0.0001 ;{ }^{* \star \star} P<0.001$. (C) Immunofluorescence staining of $\mathrm{H} 3 \mathrm{~K} 9 \mathrm{me} 3$ in the indicated regions of the hippocampus from young and old monkeys. Representative images are shown on the left; H3K9me3-positive cells are quantified as fold changes of their numbers in the old DG, CA1 and CA3 regions vs. in young counterparts, shown as means \pm SEM on the right. Red arrows indicate the H3K9me3-negative cells. Scale bars, $20 \mu \mathrm{m}$ and $10 \mu \mathrm{m}$ (zoomed-in image). Young, $n=8$; old, $n=8$ monkeys. ${ }^{* *} P<0.01$. (D) Immunofluorescence staining of HP1Y in the indicated regions of the hippocampus from young and old monkeys. Representative images are shown on the left, arrows indicate HP1y -negative cells; while HP1y-positive cells in the old DG, CA1 and CA3 regions vs. young regions are quantified as fold changes (means \pm SEM) on the right. Black arrows indicate the HP1y-negative cells. Scale bars, $20 \mu \mathrm{m}$ and $10 \mu \mathrm{m}$ (zoomed-in image). Young, $n=8$; old, $n=8$ monkeys. ${ }^{* \star} P<$ 0.01. (E) Immunofluorescence staining of LINE-1 ORF2p in the indicated regions of the hippocampus from young and old monkeys. Representative images are shown on the left; LINE-1 ORF2p-positive cells are quantified as fold changes of their numbers in the old DG, CA1 and CA3 regions vs. in young counterparts, shown as means \pm SEM on the right. Red arrows indicate the LINE-1 ORF2p-positive cells. Scale bars, $50 \mu \mathrm{m}$ and $10 \mu \mathrm{m}$ (Zoomed in image). Young, $n=8$; old, $n=8$ monkeys. ${ }^{\star \star \star \star} P<0.0001$.

et al., 2018), especially similar neurogenesis within the SGZ of the DG (Leuner et al., 2007), they represent ideal models for the study of primate hippocampal aging. On the other hand, the high complexity in its cellular composition requires the dissection of molecular mechanisms underlying hippocampal aging and cognitive decline to be performed at single-cell resolution (He et al., 2020; Zhong et al., 2020). Emerging single-cell/nucleus RNA sequencing (scRNA-seq/ snRNA-seq) techniques have unraveled the transcriptional alterations underlying the heterogeneous process of aging at cell-type-specific resolution in multiple organs ( $\mathrm{He}$ et al.,
2020; Li et al., 2020; Ma et al., 2020a, b; Wang et al., 2020a, b). Of note, snRNA-seq provides unique advantages in analyzing tissues difficult to be dissociated, such as the neuron-rich hippocampus, thus reducing the bias in cellular capture and the accompanied transcriptional artifacts (Habib et al., 2016; He et al., 2020; Ma et al., 2020a). However, snRNA-seq has not been applied to explore the cellular and molecular alterations of hippocampal aging in the primates.

In this study, we demonstrated an array of aging-associated damages in the NHP hippocampus, including genomic and epigenomic instability, loss of proteostasis, as well as increased inflammation. To explore unique cellular and molecular characteristics underlying these age-related phenotypes, we generated a high-resolution single-nucleus transcriptomic atlas of hippocampal aging in NHPs. This atlas enabled us to identify the transcriptional alterations underlying the early onset of dysregulation in adult hippocampal neurogenesis, and to unveil the contributing factors to a hostile microenvironment for neurogenesis in the aged hippocampus. Overall, our study deposited a valuable resource for the identification of new diagnostic biomarkers and potential therapeutic targets for interventions against hippocampal aging and related human neurodegenerative disorders.

\section{RESULTS}

Accumulation of aging-associated damages in the aged hippocampus from cynomolgus monkeys

To unravel the structural and functional alterations during physiological aging, we first isolated hippocampal tissues from eight young (4-6 years old) and eight aged (18-21 years old) cynomolgus monkeys, which are approximately $\sim 16$ and $\sim 60$ years old in human age, respectively (Figs. 1A and S1A) (Li et al., 2020; Ma et al., 2020b; Wang et al., $2020 \mathrm{a}$, b; Zhang et al., 2020c). We compared different anatomical regions of the hippocampus, including CA1, CA3, and the dentate gyrus (DG), and found their width and overall cell density were comparable to those in the young counterparts (Figs. 1B, 1C and S1B). However, all three regions showed an increase in cellular senescence, as the proportions of senescence-associated $\beta$-galactosidase (SA$\beta$-Gal) staining-positive cells escalated with age (Fig. 1D). In line with the notion that neurogenesis decay was usually defined as one of the hippocampal aging hallmarks (Navarro Negredo et al., 2020), we observed a decrease of DCXpositive neural progenitor cells/neuroblasts in the subgranular zone (SGZ) of the hippocampal DG (Fig. 1E) (Artegiani et al., 2017; Mauffrey et al., 2019). Moreover, we found aging-associated accumulation of a broad range of protein aggregates and amyloid- $\beta(A \beta)$ deposits (immunostained by the pan-specific anti-A $\beta$ antibody (4G8) and the anti-A $\beta 40$ antibody) (Fig. 1F-H) (Brunk and Terman, 2002; Buckig et al., 2002; Li et al., 2004; Wegiel et al., 2012; Giacobini and Gold, 2013). Importantly, we also found an accompanying increase in genomic and epigenomic instability in these 
A

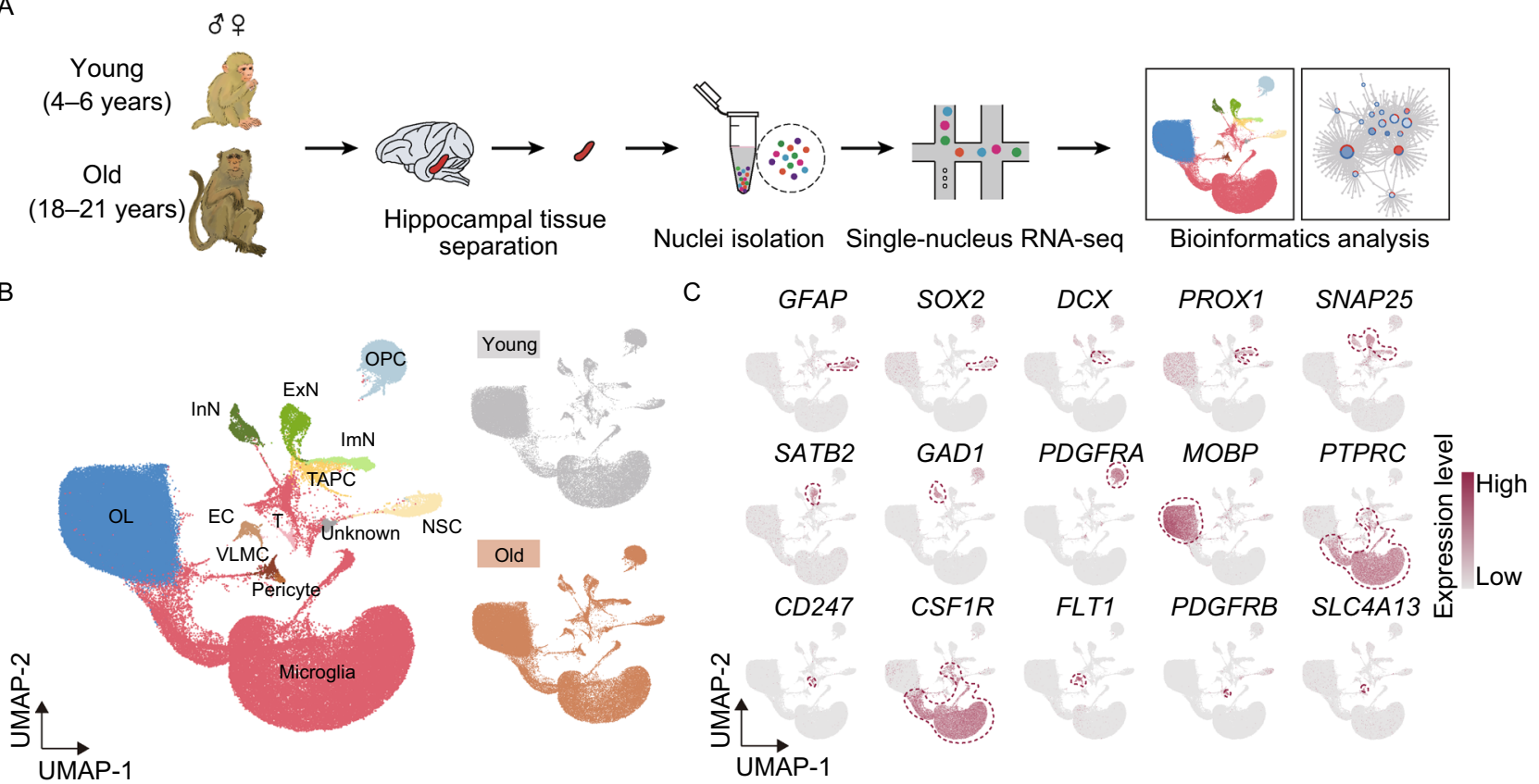

D

Top 30 marker genes

E

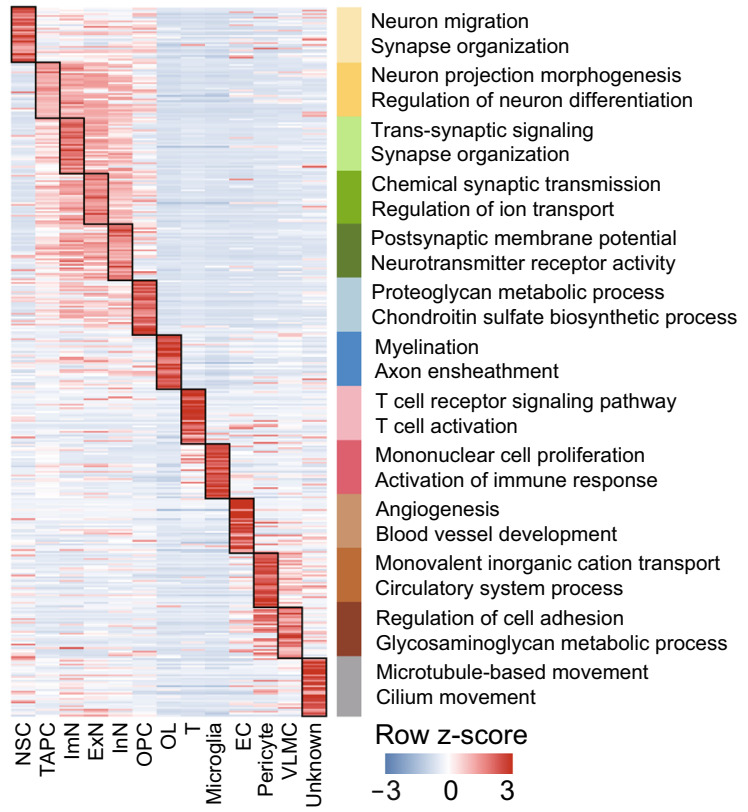

Transcriptional regulators of marker genes

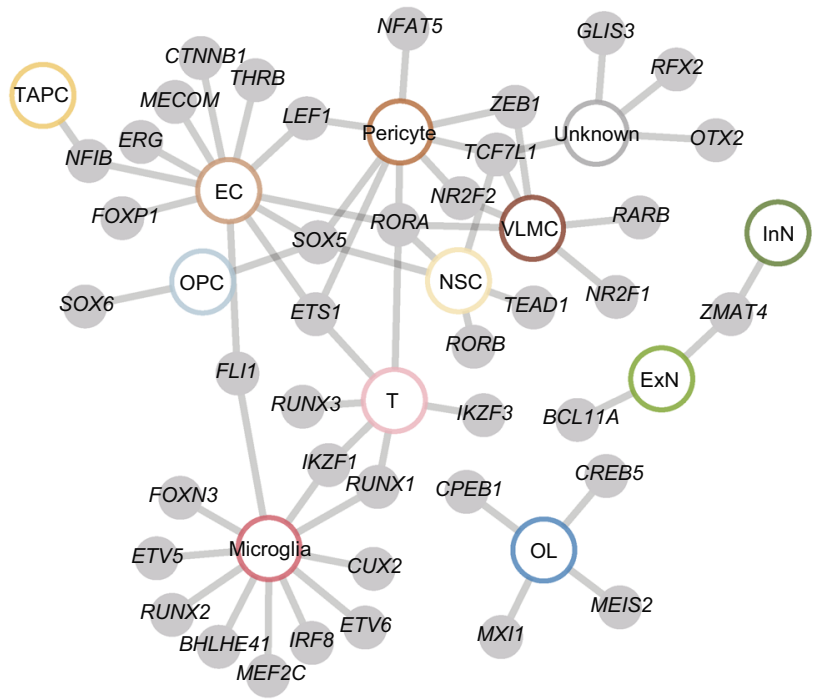

Figure 3. Construction of single-nucleus transcriptomic atlas of the monkey hippocampus. (A) Flow chart of snRNA-seq and bioinformatics analysis of the monkey hippocampus. Young, $n=7$; old, $n=8$ monkeys. (B) Left, UMAP plot showing distribution of different cell types in the monkey hippocampus. Right, UMAP plots showing distribution of different cell types in the young (top) and old (bottom) hippocampus. (C) UMAP plots showing the expression profiles of indicated cell-type-specific marker genes of corresponding cell types in the monkey hippocampus. (D) Heatmap showing the expression profiles of top 30 cell-type-specific marker genes of different cell types in the monkey hippocampus with their enriched functional annotations on the right. (E) Network plot showing transcriptional regulators of cell-type-specific marker genes (adjusted $P$-value $<0.05,|\log \mathrm{FC}|>1$ ) of different cell types in the monkey hippocampus. 

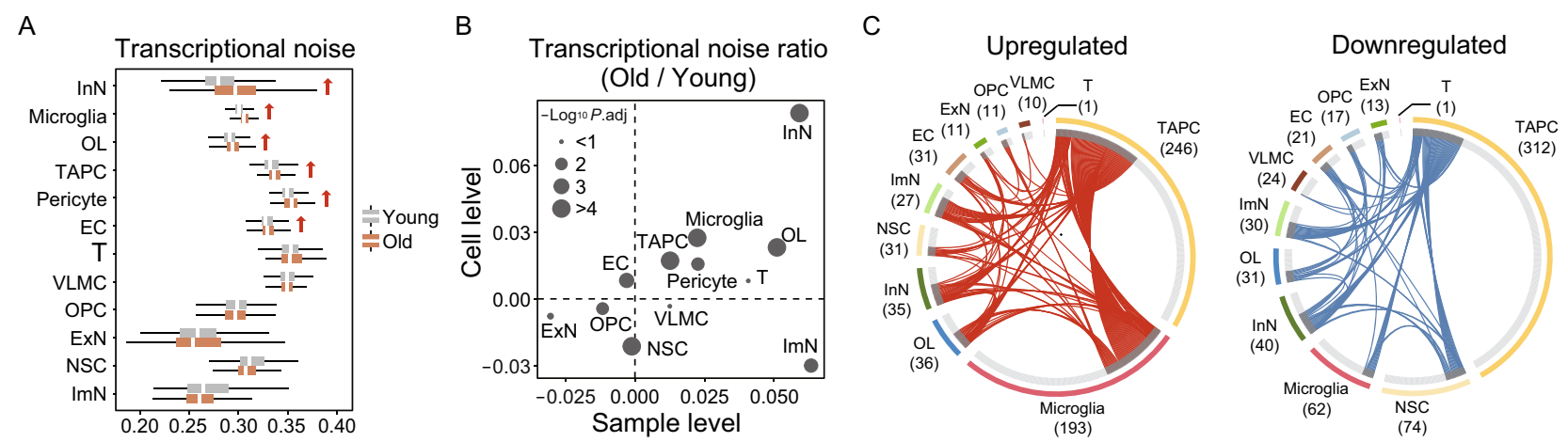

$\mathrm{D}$

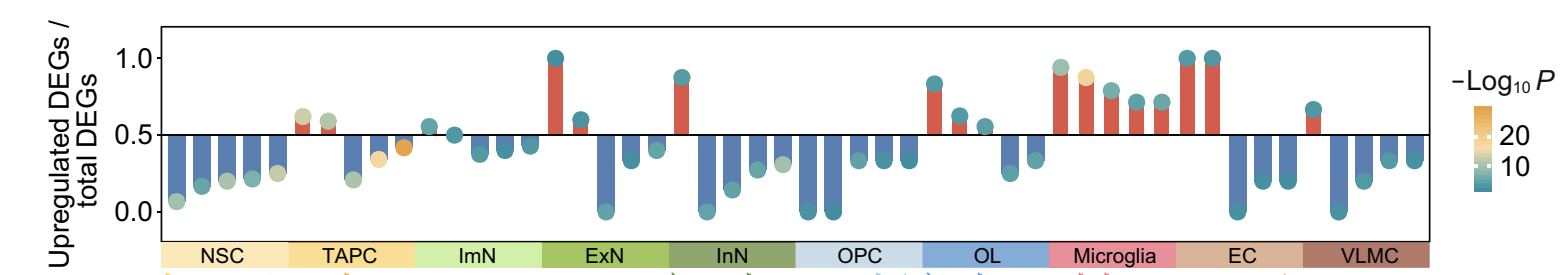

E

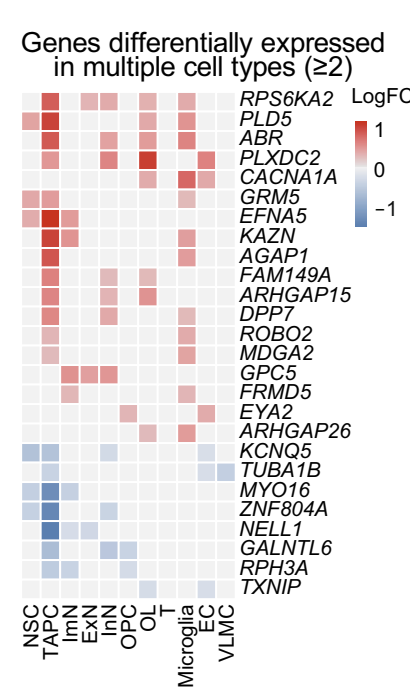

$\mathrm{F}$

Top 5 cell type-specific DEGs
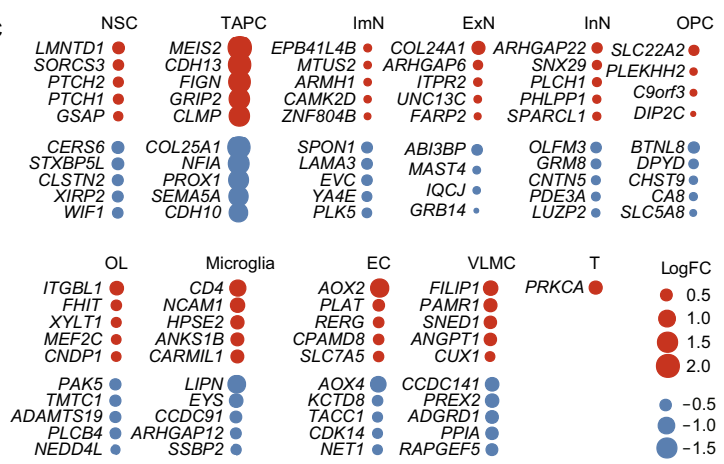

G

Figure 4. Cellular and molecular aging characteristics of the aged monkey hippocampus. (A) Boxplot showing transcriptional noise in different cell types in young and old monkey hippocampus. Arrows indicate cell types whose transcriptional noise is significantly increased in the aged groups. (B) Scatter plot showing the $\log _{2}$ ratio of transcriptional noise of different cell types in the monkey hippocampus between the old and young groups at the cell and sample levels. (C) Circos plots showing aging-related upand down-regulated differentially expressed genes (DEGs) (adjusted $P$-value $<0.05,|\log F C|>0.25$ ) of different cell types in the monkey hippocampus. Each connecting curve represents a gene that is up- or down-regulated in two cell types. (D) Bar plot showing GO terms enriched for aging-related DEGs of different cell types in the monkey hippocampus. $Y$ axis represents the ratio of upregulated genes to total DEGs in corresponding terms. (E) Heatmap showing genes differentially expressed in at least two cell types in the monkey hippocampus. Only genes with same direction of differential expression among different cell types are included. (F) Dot plots showing top five cell-type-specific DEGs of different cell types. Only those with annotations are showed. Red dots represent upregulated genes and blue dots represent downregulated ones. (G) Network plot showing DEGs associated with agingrelated diseases in different cell types in the monkey hippocampus. 
A

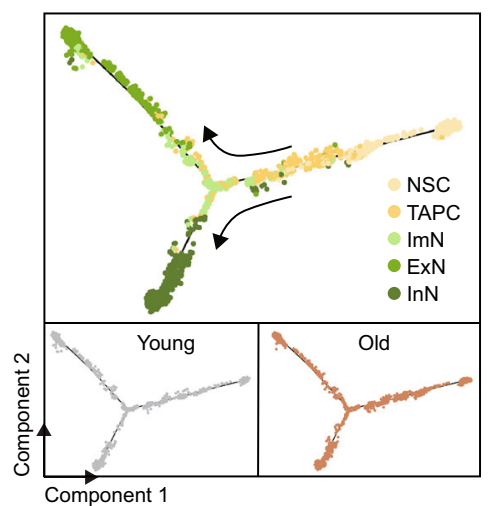

B

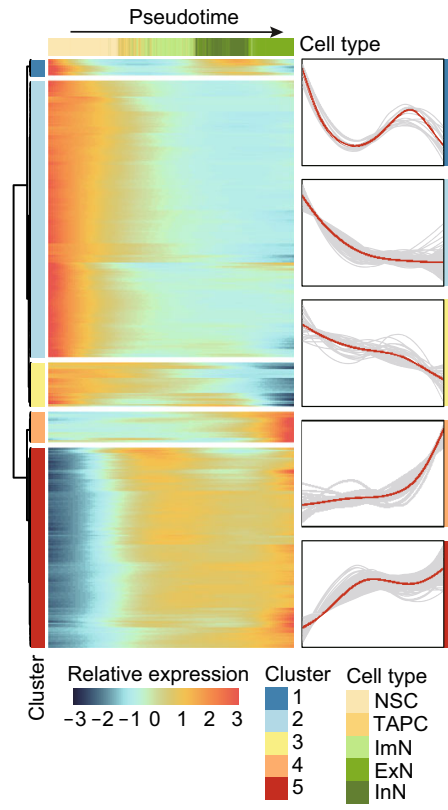

G

ZMAT4 target genes

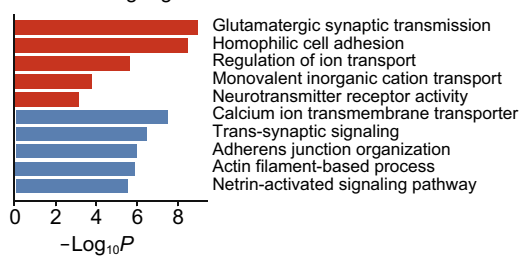

$\mathrm{H}$

NR2F2 target genes

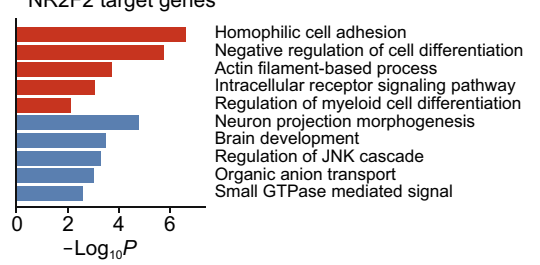

C Young Old

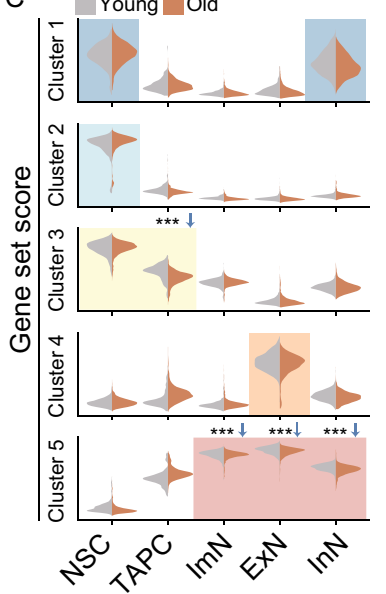

Phosphatidylinositol-mediated signaling Canonical Wnt signaling pathway Blood circulation

Response to wounding

Cell morphogenesis involved in differentiation Regulation of cell adhesion

Establishment or maintenance of cell polarity TGF-beta receptor signaling pathway

Phosphatidylinositol 3-kinase signaling

Cell fate commitment

$\mathrm{G}$ protein-coupled receptor signaling pathway Chemical synaptic transmission

Trans-synaptic signaling

Regulation of membrane potential

Regulation of ion transport
D

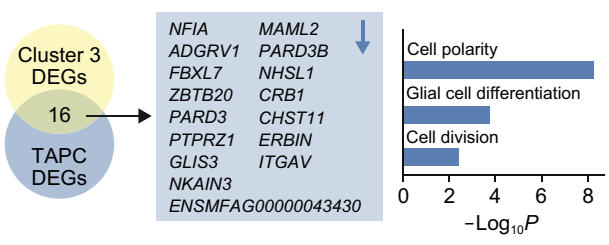

E

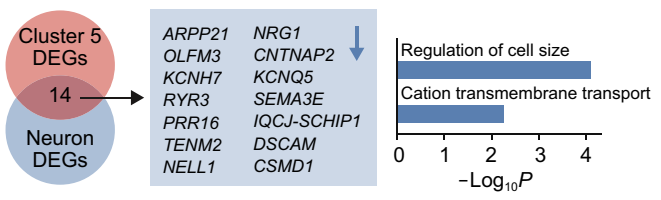

F

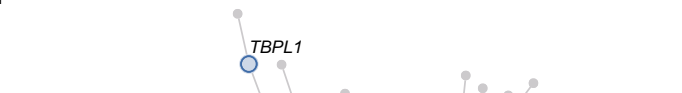

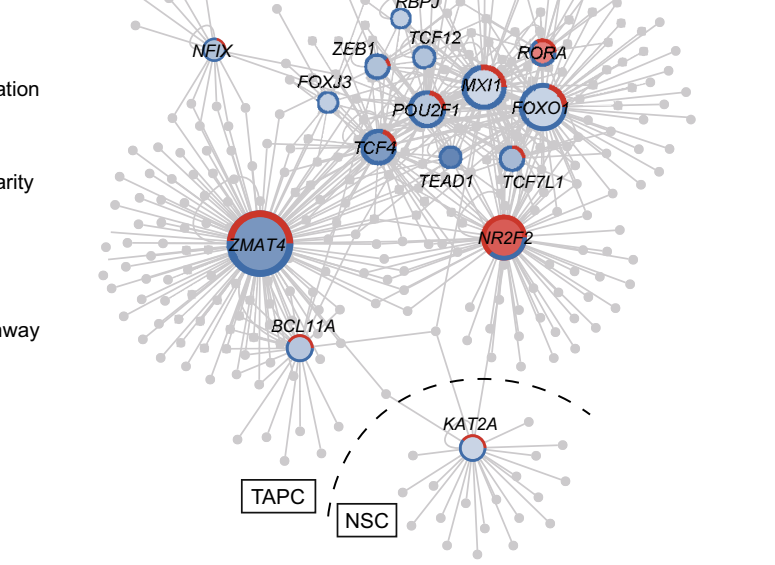

Ratio of up-/down-regulated LogFC Number of target genes

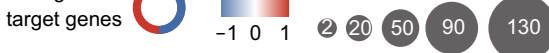

I

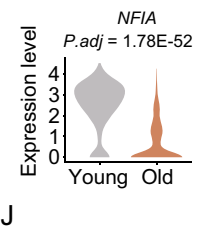
\begin{tabular}{l} 
J \\
$\begin{array}{l}\text { Correlation with gene set } \\
\text { score of cluster } 3 \mathrm{DEGs}\end{array}$ \\
\hline
\end{tabular}

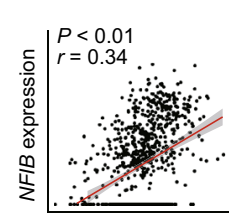

Gene set score of cluster 3 DEGs

$\mathrm{K}$

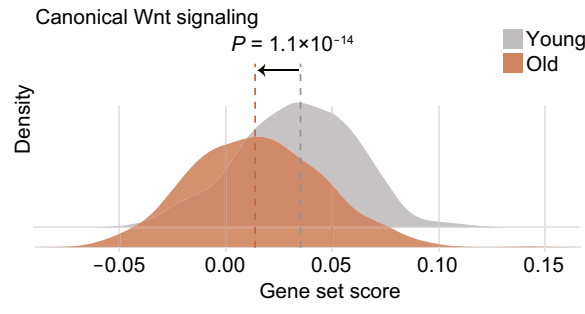

L

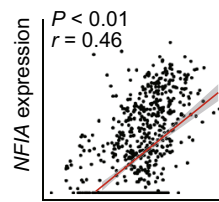

Young

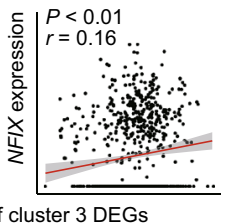

Old

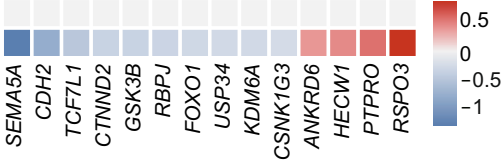


Figure 5. Aging-related cellular and molecular alterations along the trajectories of the neurogenesis. (A) Pseudotime analysis of the neurogenic lineage cells in the monkey hippocampus. The points are colored by cell types (top) and age (bottom). The arrows indicate the directions of differentiation trajectories. (B) Heatmap showing the expression profiles along the pseudotime of top 500 DEGs (q value $<1 \times 10^{-4}$ ), which were divided into five clusters with the expression pattern and enriched GO terms of the corresponding cluster represented on the right. (C) Violin plots showing gene set scores of indicated clusters in different stages of neurogenic cells of young and old groups. ${ }^{* *} P<0.01$. (D) Left, pie plot showing overlapped genes between cluster 3 DEGs and aging-related downregulated DEGs of TAPC in the monkey hippocampus. Right, bar plot showing enriched GO terms of the overlapped genes listed on the left. (E) Left, pie plot showing overlapped genes between cluster 5 DEGs and aging-related downregulated DEGs of neurons in the monkey hippocampus. Right, bar plot showing enriched GO terms of the overlapped genes listed on the left. $(F)$ Network plot showing transcriptional regulators of aging-related DEGs in NSC and TAPC in the monkey hippocampus. Node size indicates the number of target genes. Outer circle of the node indicates the proportion of up regulated (red) and down regulated (blue) target genes regulated by corresponding transcriptional regulators. (G) Bar plot showing GO terms of target genes of ZMAT4. Red, upregulation; blue, downregulation. $(\mathrm{H})$ Bar plot showing $\mathrm{GO}$ terms of target genes of NR2F2. Red, upregulation; blue, downregulation. (I) Violin plots showing expression levels of NFIA, NFIB and NFIX in TAPC in the monkey hippocampus from young and old groups. (J) Spearman's correlations between gene set score of cluster 3 DEGs and the expression levels of NFIA, NFIB and NFIX in TAPC of the monkey hippocampus. (K) Density plot showing gene set scores of genes related to canonical Wnt signaling pathway in TAPC of the monkey hippocampus from young and old groups. (L) Heatmap showing the expression changes of aging-related DEGs associated with canonical Wnt signaling in TAPC of the monkey hippocampus from young and old groups.

regions, such as increased DNA damage (marked by $\mathrm{y}-\mathrm{H} 2 \mathrm{~A}$. $X$ foci formation), increased cytosolic dsDNA release from the nucleus, loss of heterochromatin (marked by H3K9me3 and $\mathrm{HP} 1 \gamma$ reduction) and retrotransposon activation (marked by LINE-1 ORF2p accumulation) (Fig. 2A-E) (De Cecco et al., 2019; Geng et al., 2019; Hu et al., 2020; Ma et al., 2020a; Zhang et al., 2020b). Altogether, these results supported that the NHP hippocampus suffered from extensive aging-associated damages during physiological aging even without obvious overall structural disorganization.

\section{Construction of a single-nucleus transcriptomic atlas of the NHP hippocampus}

To investigate the cellular and molecular signatures underlying hippocampal aging, we applied the snRNA-seq technique to capture all cell types in the hippocampus. After stringent filtration, 116,951 qualified single-nucleus transcriptomes were retained with a median of 1,972 genes per nucleus from young and old individuals for downstream analysis (Figs. 3A, 3B and S1C). No apparent difference in cell type distribution was observed among age and sex groups (Fig. S1D), guaranteeing the reliability of gene expression profiling. Uniform Manifold and Projection (UMAP) algorithm analysis identified a total of 12 cell types based on their unique gene-expression signatures (Fig. 3B, $3 \mathrm{C}$ and Table S1). Consistent with previous reports (Boldrini et al., 2018), the hippocampal cell populations were constituted by three major cell types, including neurogenic lineage cells, oligodendrocyte lineage cells and niche cells. Neurogenic lineage cells include neural stem cells (NSC) (GFAP and $S O X 2^{+}$), transiently amplifying progenitor cells (TAPC) $\left(D C X^{+}, P R O X 1^{+}\right.$and $\left.S N A P 25^{-}\right)$, immature neurons $(\operatorname{ImN})$ $\left(P R O X 1^{+}\right.$and $\left.S N A P 25^{+}\right)$, excitatory neurons (ExN) $\left(S A T B 2^{+}\right.$ and $\left.S N A P 25^{+}\right)$, and inhibitory neurons $(\operatorname{InN})\left(G A D 1^{+}\right.$and $S N A P 25^{+}$) (Fig. $3 B$ and $3 C$ ). Oligodendrocyte lineage cells comprise two cell types, the oligodendrocyte progenitor cells (OPC) (PDGFRA ${ }^{+}$), and oligodendrocytes (OL) $\left(\mathrm{MOBP}^{+}\right)$ (Fig. 3B and $3 \mathrm{C}$ ). As for niche cells, they were composed of T cells $(T)\left(C D 247^{+}\right)$, microglia $\left(C S F 1 R^{+}\right)$, endothelial cells (EC) $\left(F L T 1^{+}\right)$, pericytes $\left(P D G F R B^{+}\right)$, vascular leptomeningeal cells (VLMC) (SLC4A13 ${ }^{+}$) (Fig. 3B and 3C). In addition to canonical markers, we also identified a set of novel markers for each cell type (Table S2). Gene Ontology (GO) analysis of these cell-type-specific marker genes demonstrated functional characteristics of the corresponding cell type in the hippocampus (Fig. 3D and Table S1). For example, "Neuron migration" was enriched for the top 30 marker genes for NSC, "Regulation of neuron differentiation" for TAPC, "Synapse organization" for ImN, "Chemical synaptic transmission" for ExN, "Postsynaptic membrane potential" for InN, "Proteoglycan metabolic process" for OPC, and "Myelination" for OL, etc.

Moreover, we constructed a regulatory network of transcription factors (TFs), which defined core TFs unique for each cell type and hub TFs shared by at least two cell types (Fig. 3E and Table S1). For instance, the prominent genes comprising the cell-type-specific TF network included TEAD1 for NSC, SOX6 for OPC, CREB5 for OL, and so on (Fig. 3E). Meanwhile, ZMAT4 was shared by ExN and $\operatorname{InN}$, suggesting its essential roles in neuron specification and functional maintenance. SOX5 was shared by NSC, OPC, EC and pericyte, indicative of its function as a broad-acting transcriptional regulator. The network depicted the unique and coordinated transcriptional regulations for the establishment of hippocampal cell identities. Taken together, this atlas allows for, in our following analysis, a comprehensive delineation of the cellular and molecular alterations induced by aging in the NHP hippocampus. 
A

Upregulated DEGs of microglia

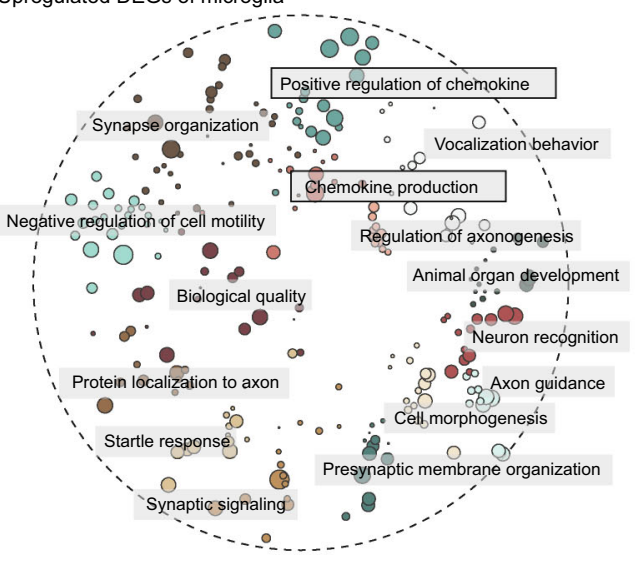

E

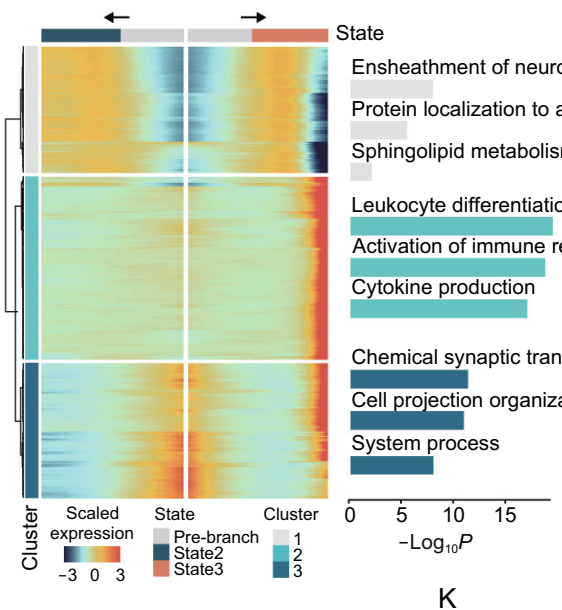

I

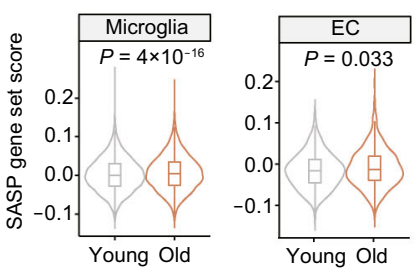

J

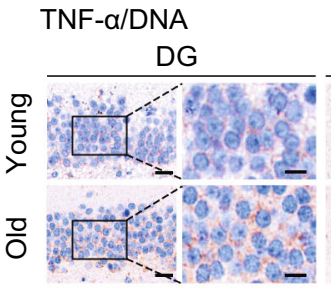

Downregulated DEGs of microglia
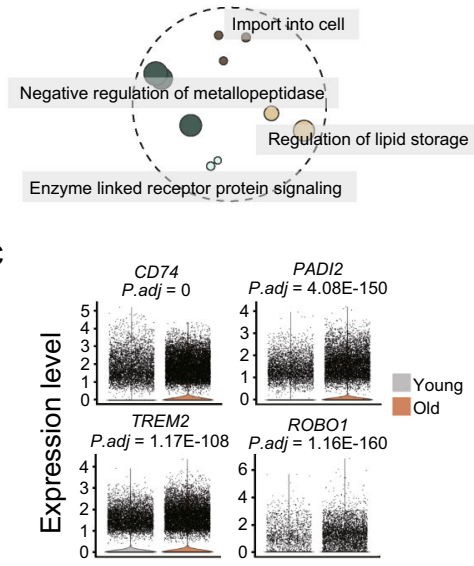

F

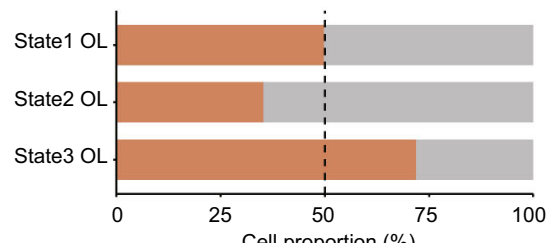

G

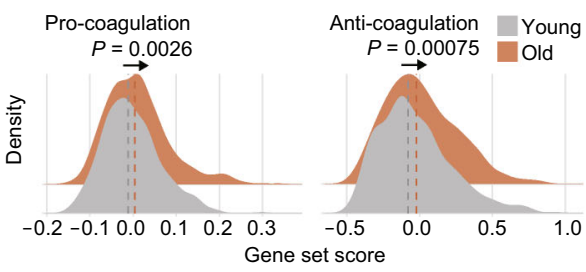

L

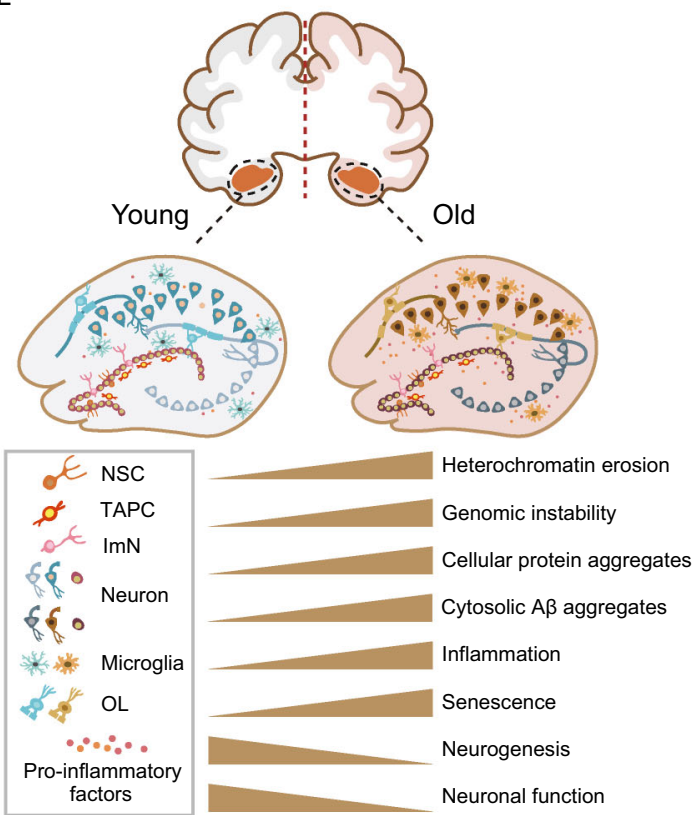

D
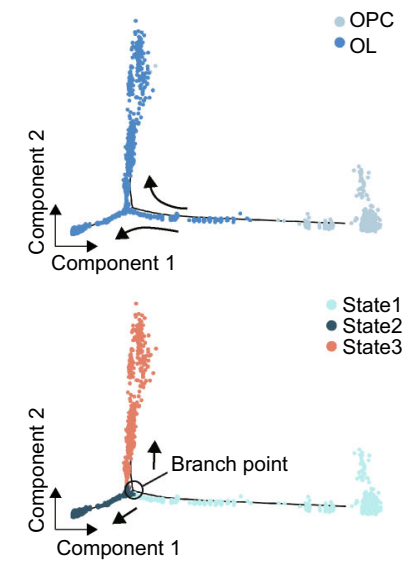

$\mathrm{H}$

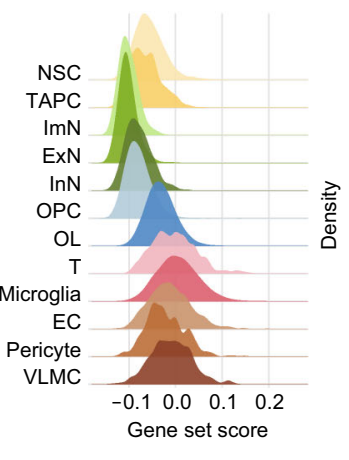

LogFC

1
0.5
0

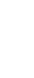

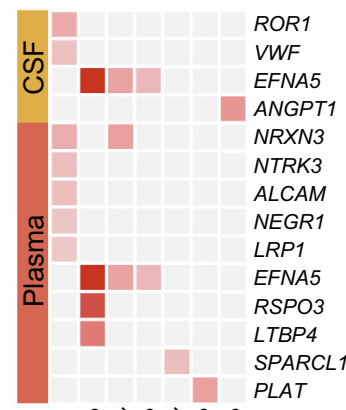

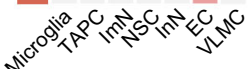

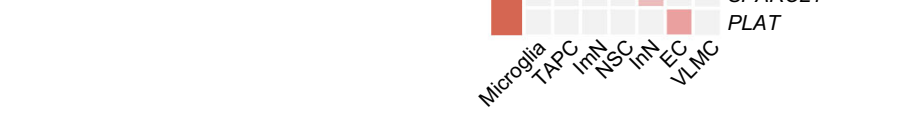


Figure 6. Elevated inflammation with age in the monkey hippocampus. (A) Dot plot showing GO terms of aging-related upregulated DEGs in microglia of the monkey hippocampus. Each dot indicates a GO term and similar entries were clustered together. (B) Dot plot showing GO terms of aging-related downregulated DEGs in microglia of the monkey hippocampus. Each dot indicates a GO term and similar entries were clustered together. (C) Violin plots showing expression levels of indicated genes in microglia of the monkey hippocampus from young and old. (D) Pseudotime analysis of OPC and OL in the monkey hippocampus. Cells are colored by the cell types (top) and the states (below). The arrows indicate the directions of differentiation trajectories. (E) Heatmap showing the expression profiles along the pseudotime of top 500 DEGs (q value $<1 \times$ $10^{-4}$ ) in OL and OPC, which were then divided into three clusters with the expression pattern and enriched $\mathrm{GO}$ terms of the corresponding cluster represented on the right. $(F)$ Bar plot showing the proportions of different states of $\mathrm{OL}$ in the hippocampus from young and old groups. (G) Density plot showing gene set scores of pro-coagulation and anti-coagulation genes in EC of the monkey hippocampus from young and old groups. (H) Density plot showing gene set scores of senescence-associated secretory phenotype (SASP) genes in different cell types in the monkey hippocampus. (I) Violin plots showing increasing SASP gene set scores in microglia and EC in the monkey hippocampus from young and old groups. (J) Immunohistochemical staining of TNF- $\alpha$ (brown) and counterstaining of cell nuclei by hematoxylin solution (blue) in the hippocampus from young and old monkeys. Representative images are shown on the left; signal intensity of TNF- $\alpha$ is quantified as fold changes in the old DG and CA1 regions vs. in young counterparts, shown as means \pm SEM on the right. Scale bars, $20 \mu \mathrm{m}$ and $10 \mu \mathrm{m}$ (zoomed-in images). Young, $n=8$; old, $n=8$ monkeys. ${ }^{* \star *} P<0.0001$. (K) Heatmap showing agingrelated DEGs and their age-associated protein products in human cerebrospinal fluid (CSF) and plasma in different cell types in the monkey hippocampus. (L) A schematic illustration showing the phenotypic and transcriptomic signatures of NHP hippocampal aging.

Characterization of the aging-associated cellular and molecular profiles of the NHP hippocampus

To dissect cell-type-specific aging-related alterations in gene expression, we first examined gene expression signatures of marker genes for each cell type in the NHP hippocampus. The unchanged expression signatures suggested that cell identities remain relatively stable during aging (Fig. S2A). Yet, given the profound genomic and epigenomic destabilization observed in various hippocampal regions (Fig. 2), we wondered if there are similar transcriptional instabilities with age. As expected, calculation of the transcriptional noise indicated that TAPC and $\ln N$ in the neurogenic lineage cells, $\mathrm{OL}$ in the oligodendrocyte lineage cells, as well as microglia, pericyte and EC in the niche cells exhibited higher transcriptional noise with age (Fig. $4 \mathrm{~A}$ and $4 \mathrm{~B}$ ), which was in agreement with the increased transcriptional noise observed in other aged tissues (Li et al., 2020; Wang et al., 2020a; Zhang et al., 2020c). In addition, to annotate the hotspot genes involved in aging and age-related diseases, we performed an integrative comparative analysis between celltype-specific marker genes and genes from the Aging Atlas (AA) gene set and gene sets implicated in learning and memory disorders (LD, MD) as well as neurodegenerative diseases including Alzheimer's disease (AD) and Parkinson's disease (PD) (Aging Atlas, 2021). The marker genes of microglia and EC were the most frequently annotated as hotspot genes (Fig. S2B), indicating that these cell types may be more susceptible to aging and age-related diseases.

In order to further identify the critical cell types affected by and/or responsible for hippocampal aging, we analyzed ageassociated differentially expressed genes (DEGs) across all cell types. The largest numbers of DEGs were observed in TAPC, microglia, and NSC (246, 193 and 31 upregulated genes, 312, 62 and 74 downregulated genes, respectively in aged tissues) (Fig. 4C and Table S2). In addition, the numbers of DEGs in the male group were larger than their counterparts in the female group across almost all cell types (Fig. S2C), suggesting that the male hippocampus may be more susceptible to aging. Further, through functional annotation enrichment analysis, we investigated molecular pathways most affected by aging in these cell types. We noticed that "neuron differentiation" was enriched for downregulated DEGs in both $\mathrm{NSC}$ and $\operatorname{ImN}$, suggesting for the early onset of dysregulation of genes related to neurogenesis. Secondly, increased expression of genes involved in "cell junction organization" and "actin filament-based process" in ExN (Fig. 4D) implied for the correlation between a dysregulation of the cytoskeleton and compromised synaptic dynamics with age (Végh et al., 2014). Also contributing to age-related synaptic degeneration are oligodendrocyte lineage cells, as GO analysis of DEGs in OPC and $O L$ revealed increased "apoptotic process", along with decreased "neuron recognition" and "synaptic transmission" (Fig. 4D). Lastly, for niche cells, increased "chemokine production" and "receptor-mediated endocytosis" in microglia, together with the elevated "zymogen activation" and "endothelial cell migration" in EC and VLMC (Fig. 4D), pointed to elevated inflammation and debris engulfment along with compromised supporting abilities with age.

In addition, a total of 18 genes were consistently upregulated, along with 8 consistently downregulated ones in at least two cell types (Fig. 4E). For instance, ROBO2, which encodes roundabout guidance receptor 2 and promotes proinflammatory immune response (Martinelli and Real, 2019), is upregulated in aged TAPC and microglia (Fig. 4E), suggesting elevated inflammation in the aged hippocampus (Fig. 4D). NELL1, which encodes a cytoplasmic protein that contains epidermal growth factor (EGF)-like repeats and is involved in cell growth regulation and differentiation (Nakamura et al., 2012), is downregulated in TAPC and derivative 
neurons (Fig. 4E), correlative with the aberrant neurogenesis. On the other hand, for cell-type-specific DEGs, we listed the top 5 genes for each cell type and noticed that their dysregulation might compromise corresponding cellular functions (Fig. 4F and Table S2). For instance, GSAP, an upregulated gene in aged NSC, was reported to be responsible for promoting amyloid-beta $(A \beta)$ production through interaction with both gamma-secretase and its substrate (He et al., 2010), thus probably contributing to the increased $A \beta$ deposits observed in the aged hippocampus (Fig. 1F). CDH13, upregulated in aged TAPC, encodes a calcium-dependent cell adhesion protein with a central role in the regulation of brain network development and synaptic plasticity, and hence is associated with neuropsychriatric disorders (Rivero et al., 2013). GRM8, downregulated in aged $\mathrm{InN}$, encodes a glutamate metabotropic receptor of neurotransmitter L-glutamate, and is known to facilitate neuronal resilience to central nervous system (CNS) inflammation (Woo et al., 2021). PAK5 was downregulated in OL, which may impair cytoskeletal remodeling, and compromise the synaptic vesicle trafficking and axon-myelin contacts (Zhang et al., 2020a). These dysregulated genes may underlie the progressive functional decay of hippocampal cells during aging.

Next, we performed an integrative comparative analysis between newly identified age-associated DEGs of the NHP hippocampus and annotated hotspot genes from various human neurodegenerative disease-associated gene sets or from the Aging Atlas gene set (Fig. 4G) (Aging Atlas, 2021). Through the construction of a high-risk DEG network linking hippocampal aging and neurodegenerative diseases, we found that most of the high-risk DEGs were enriched in TAPC and microglia. This network suggested that these two cell types were more susceptible to age-related diseases, especially for $A D$, as exemplified by TREM2, INPP5D, SORL1, etc. (Fig. 4G). Among them, TREM2 encodes an essential microglia sensor that mediates their response to environmental signals (Leyns et al., 2017), and its upregulation in microglia is closely related to inflammatory responses and pathogenesis of AD (Keren-Shaul et al., 2017; Ulland and Colonna, 2018). Meanwhile, some high-risk DEGs were associated with multiple disorders (Fig. 4G). For example, $P R K N$ is a risk factor for both learning and memory disorders, as well as PD, as it encodes a component of a multiprotein E3 ubiquitin ligase complex (Lubbe et al., 2021), whose upregulation may be linked to the misregulation of proteostasis in the aged hippocampus (Fig. 1F) (CostaMattioli and Walter, 2020). Among these high-risk genes, those dysregulated in more than one cell type, such as CNTNAP2 and SORL1 (Fig. 4G), may represent potential targets to defer the onset of cognitive decline and neurodegenerative diseases in the elderly.

In addition to intrinsic changes, direct cell-to-cell contacts also modulate neurogenesis during aging (Ransohoff, 2016; Leng and Edison, 2021), which may precede the decline in neurogenesis. Thus, we preformed cell-cell interaction analysis across all cell types and discovered a global increase in cell-cell interactions in the aged hippocampus compared to the young counterparts (Fig. S2E, S2F and Table S3), which was consistent with the observations in other aged tissues (Ma et al., 2020a, b). Of note, VLMC, TAPC, and OPC were the top three cell types that display the most old-specific cell-cell interactions; while TAPC, EC and pericyte were the top three cell types that demonstrate the most young-specific interactions (Fig. S2E and S2F), pinpointing the aberrant communications between the neural progenitor cells and neurogenic niche cells, which may contribute to the compromised neurogenesis.

Altogether, our findings identified aging-related molecular features of the NHP hippocampus, demonstrating impaired neurogenesis and neuronal function, increased inflammatory response as the most affected and potential hallmarks in the age-related neurodegeneration of the hippocampus.

Profiling of aging-related molecular alterations along the differentiation trajectories in hippocampal neurogenesis

To determine the molecular disruptions underlying impaired neurogenesis with age, we first inferred the differentiation trajectories of NSC in the hippocampal neurogenesis, starting from NSCs, through sequential and stepwise developmental progenitor cells, immature neurons, and finally to mature neurons, as indicated by pseudotime analysis (Figs. 5A and S3A), and observed no obvious difference of cell-type distribution along the trajectories between the young and old groups (Fig. 5A). Also, we established the underlying molecular cascades with respect to the pseudotime. For example, the differential expression of representative maker genes reflected the transition from quiescence to activation of NSCs, then proliferation, and differentiation into neurons (Fig. S3B). After clustering of stage-specific gene expression along the pseudotime, we obtained five distinct clusters of expression profiles and analyzed their corresponding enriched GO terms (Fig. 5B and Table S4). For example, cluster 3 defined genes progressively downregulated with the trajectories, which were enriched in pathways regulating "establishment of maintenance of cell polarity" (Fig. 5B). By contrast, cluster 5 defined genes progressively upregulated along the trajectories, and signature genes were involved in "trans-synaptic signaling" and "regulation of ion transport" (Fig. 5B).

Next, to investigate age-related alterations along the trajectories during neurogenesis, we compared the expression profiles of cluster-specific genes between the young and the old (Fig. 5C). We observed significant downregulations of cluster 3-specific genes in aged TAPC and of cluster 5 -specific genes in aged neurons (Fig. 5C). Notably, by joint analysis of cluster-specific DEGs and age-related DEGs, we found 16 downregulated genes enriched for "cell polarity" and "cell division" in TAPC (NFIA, MAML2, etc.) (Fig. 5D), 
reminiscent of the reduced proportion of S-phase TAPC in the aged hippocampus and decreased DCX-positive progenitor cells in the DG region (Figs. $1 E$ and $S 3 C$ ). And 14 downregulated genes shared between cluster 5 -specific DEGs and aged neuron-specific DEGs (ARPP21, NRG1, etc.) were enriched for "cation transmembrane transport" (Fig. 5E), indicating the possibility of dysfunction in neurons of the aged hippocampus. Collectively, these results suggested aberrant neurogenesis in the aged hippocampus, including compromised TAPC proliferation at the initial stage and impaired neuron functions at the late stage of neurogenesis.

Then we set out to explore the transcriptional regulatory network governing age-associated DEGs of neuronal lineage cells. Of note, the results revealed a panel of key transcription factors (TFs), such as ZMAT4 (Zinc Finger Matrin-Type 4), NR2F2 (Nuclear Receptor Subfamily 2 Group F Member 2), NFIX (Nuclear Factor I X), etc. in TAPC and KAT2A (Lysine Acetyltransferase 2A) in NSC (Fig. 5F and Table S5), which regulated the expression of a set of genes associated with memory and learning (Fig. S3D). Specifically, GO enrichment analysis of genes targeted by ZMAT4 and NR2F2, respectively, pinpointed that these two major hub TFs regulated a series of events involved in neurogenesis and neuronal synaptic signaling (Fig. 5G and $5 \mathrm{H})$. Interestingly, we also noticed a consistent downregulation of Nuclear Factor I (NFI) family members including NFIA, NFIB, NFIX in aged TAPC (Fig. 5D, 5F and 5I). Combined with a correlation analysis, we demonstrated a positive correlation of NFIX family members with cluster 3-specific genes (Fig. 5J), which are highly expressed at the early stage of the neurogenesis trajectory. This suggests a prominent role of NFI family TFs in the initiation of TAPC differentiation, whose downregulation may contribute to an aberrant transcriptional regulatory network and compromise the initial stages of neurogenesis. Other key TFs, such as TCF7L1 and TCF12 (Fig. 5F), were core TFs involved in the Wnt signaling pathway. Therefore, we performed the gene set score analysis for the canonical Wnt signaling pathway (Fig. 5K). As expected, we observed a decrease of the gene set score in the aged samples, reflected by an upregulation of inhibitory genes in Wnt signaling including ANKRD6 and $H E C W 1$, as well as a downregulation of core activating genes such as CTNND2 (Fig. $5 \mathrm{~L}$ ). As Wnt signaling is essential for NSC activation and TAPC differentiation (Bengoa-Vergniory and Kypta, 2015), its dysregulation further supported the compromised neurogenesis during hippocampal aging.

Altogether, dysregulation at multiple steps along the neurogenesis trajectory of the aged hippocampus, especially at the initial stage of neurogenesis and at the later stage of synaptic function, constituted one of the major alterations in the aged NHP hippocampus and may contribute to impaired cognition.
Identification of elevated neuroinflammation in aged NHP hippocampus

In addition to intrinsic changes, neurogenesis is profoundly influenced by the neurogenic niche, which is comprised of microglia, oligodendrocytes, neurovasculature, and the systemic cytokine and chemokine environment (Kempermann et al., 2015). It has been reported that neuroinflammation is a known negative regulator of adult hippocampal neurogenesis (Fan et al., 2017; Jin et al., 2021; Leng and Edison, 2021), in which age-induced microglia activation and proinflammatory factors play a central role (Leng and Edison, 2021). Through analysis of age-related DEGs, we observed that upregulated genes were enriched for "regulation of chemokine" and "chemokine production" in the aged cells (Fig. 6A and 6B). Moreover, a panel of genes related to microglia activation, i.e., CD74, TREM2, PADI2, ROBO1, were upregulated in the aged microglia compared to their younger counterparts (Fig. 6C). For example, the established marker for activated M1 microglia CD74 acts as a cellsurface receptor for macrophage migration inhibitory factor (MIF) and a multifunctional trigger for the inflammatory response (Hwang et al., 2017; Su et al., 2017). TREM2 contributes to the age-related microglial activation, the phagocytic oxidative burst, and the loss of neurons with possible detrimental effects during physiological aging, and were recently found to increase the risk for developing $A D$ (Leyns et al., 2017; Linnartz-Gerlach et al., 2019). PADI2 is known to promote the production of pro-inflammatory factors $\mathrm{IL}-1 \beta, \mathrm{IL}-6$, and TNF- $\alpha$ in macrophages, and has been implicated in chronic inflammatory diseases (Wang et al., 2017; Yu et al., 2020). SLIT2-ROBO1 signaling also associates with the regulation of genes involved in pro-inflammation (Geutskens et al., 2010; Tiensuu et al., 2019).

Oligodendrocyte, a type of neuroglia that produces myelin, an insulating sheath on the axons of nerve fibers, constitutes an important part of the microenvironment for neurogenesis (Marques et al., 2016). We next traced the aging-related molecular changes along their developmental trajectory and reconstructed the sequential developmental steps by pseudotime analysis (Figs. 6D and S4A). Differential expression of representative marker genes demonstrated the transition from OPC to OL (Fig. S4B). After clustering of stage-specific gene expression along the pseudotime, we obtained three distinct clusters of expression profiles and their corresponding gene sets (Fig. $6 \mathrm{E}$ and Table S4). Through GO term analysis, we found that the gene set 1 was enriched for genes responsible for "ensheathment of neurons", gene set 2 was enriched for genes involved in "leukocyte differentiation" and "activation of immune response", and gene set 3 was enriched for genes regulating "chemical synaptic transmission" (Fig. 6E). Interestingly, gene set 2 is specifically expressed in an OL subtype (state $3 \mathrm{OL}$ ), whose population expanded at the advanced age (Figs. 6F and S4C). Collectively, these findings suggest an age-associated activation of inflammation 
and consequent promotion of a pro-inflammatory microenvironment impairing neurogenesis.

The neurovasculature is a critical node connecting the neurogenic niche with the systemic environment, whose dysregulation has been proposed to impair neurogenesis. As aforementioned, we found increased zymogen activation in the aged ECs (Fig. 4D), including PLAT, a plasminogen activator required for fibrinolysis (Kruithof and DunoyerGeindre, 2014), whose upregulation may result in the formation of hemolysis in hippocampus. However, when we evaluated the expression of coagulation-related genes in ECs, we found that gene set scores of both pro-coagulation and anti-coagulation pathways were higher in aged ECs compared with those in their young counterparts (Fig. 6G), implicating the imbalance and dysregulation of coagulation and hemolysis. As coagulation and hyperfibrinolysis are closely related to disruption of blood-brain barrier permeability (Marcos-Contreras et al., 2016; Gust et al., 2017), our results suggest a plausible blood-brain barrier dysfunction involved in hippocampal aging.

We observed increased senescent cells in various regions of the aged hippocampus (Fig. 1D). As senescenceassociated secretory phenotype (SASP) is a common feature of senescent cells and usually contributes to a lowgrade inflammatory state (Baker and Petersen, 2018), we next asked if the neurogenic niche in the aged hippocampus presents elevated SASP and systemic pro-inflammatory environment. Accordingly, we noticed that various niche cells exhibited much higher gene set scores for SASP overall (Fig. 6H). Especially, for microglia and EC, the two cell types that constitute the blood-brain barrier (Vanlandewijck et al., 2018), we found elevated SASP scores with age (Figs. 6I and S4D). Our findings may reflect a rise in age-related inflammation responses observed in these two cell types and a plausible destruction of the hippocampal blood-brain barrier with age (Sweeney et al., 2018; Yang et al., 2020). Consistently, we discovered an increase in pro-inflammatory factors (Tilstra et al., 2011), represented by age-related elevation of tumor necrosis factor-alpha (TNF- $\alpha$ )-positive cells (Figs. 6J and S4E). Moreover, we used joint analysis of the upregulated genes in the NHP hippocampus with proteomic data from aged human cerebrospinal fluid (CSF) or plasma samples to pinpoint the conserved alteration in protein products in primates (Baird et al., 2012; Tanaka et al., 2018). We found that microglia harbor the highest number of conserved changes, including ROR1 and $V W F$ in the aged CSF, and NRXN3, NTRK3 and ALCAM1 in the plasma from the elderlies, respectively, providing the potential for microglia-secreted factors to serve as circulatory hallmarks in the elderlies (Fig. 6K).

To sum up, these findings indicated a pro-inflammatory neurogenic niche and underlying molecular features during hippocampal aging, suggesting the formation of a deleterious microenvironment in the primate hippocampus at an advanced age, which may exacerbate age-related neurodegeneration and facilitate the progression of neurodegenerative diseases.

\section{DISCUSSION}

To date, the cellular and molecular mechanisms underlying primate hippocampal aging have not been determined. In this study, we systemically depicted the aging-associated phenotypes, and found that NHP hippocampus underwent extensive aging-related damages during physiological aging without obvious structural disorganization. Next, we established the first primate single-nucleus transcriptomic roadmap of hippocampal aging. According to their unique transcriptional signatures, we identified 12 distinct cell types, comprised of the neurogenesis lineage, oligodendrocyte lineage, and the niche cells. Aging-related DEG analysis unraveled that TAPC and microglia were the most susceptible cell types to aging as they manifested the most DEGs, including genes annotated as high-risk genes for neurodegenerative diseases. In-depth analysis of the dynamic geneexpression signatures of the stepwise neurogenesis trajectory revealed the impaired TAPC division and compromised neuronal function as prominent aging features. The elevated pro-inflammatory response was observed in the aged microglia, OL and EC, which may trigger the formation of an inflammaging microenvironment in the primate hippocampus. Furthermore, dysregulation of coagulation-regulating genes in aged EC suggested perturbance of the blood-brain barrier integrity as a potential factor associated with hippocampal degeneration. In summary, our analyses provided a wealth of resources for understanding the aging phenotypic changes and the underlying mechanisms of the primate hippocampal aging, and shed light on the development of novel diagnostic and therapeutic interventions against agerelated neurodegenerative disorders (Fig. 6L).

Age-associated impairments of neurogenesis in DG of the hippocampus are associated with various neurodegenerative disorders (Morrison and Baxter, 2012; Fan et al., 2017; Hou et al., 2019), making hippocampus a crucial and attractive subject for both fundamental research and translational neurobiology. As a highly heterogeneous tissue, the complexity of hippocampal aging needs to be unveiled the single-cell level. A pioneering study at single-cell resolution has been carried out in the mouse hippocampus specifically for the neurogenic niche, thus only focusing on the nonneuronal cell types. They focused more on the abundance of different cell types rather than their molecular profiles due to the limitation of available high-quality single cell transcriptomes in this early study (Artegiani et al., 2017). On the other hand, a following study supporting age-associated alterations in gene expression of neuronal cells has been reported for the neural stem/progenitor cell (NSPC) subpopulations by single-cell RNA-seq in the mouse brain, although it focused on NSPC localized in the subependy$\mathrm{mal} /$ subventricular zone (SEZ/SVZ) of the forebrain, rather than those in the SGZ of the hippocampus (Shi et al., 2018). 
Yet another very recent study reported that NSC subpopulations exhibited aging-related asynchronous depletion accompanied by increased quiescence, and revealed a set of molecular hallmarks of aging in mouse hippocampus via single-cell transcriptomic analysis (Ibrayeva et al., 2021). All these previous studies advanced our understanding of agerelated neurodegeneration, although we need to be cautious applying it to human diseases due to the drastic difference in physiological structure and functions between mouse and primate brains (Herculano-Houzel, 2009; Zhang et al., 2018; Zhong et al., 2020). Therefore, a high-resolution and comprehensive study to help understand age-related molecular changes in the primate hippocampus is of pressing demand.

With recent advances of snRNA-seq technologies ( $\mathrm{He}$ et al., 2020; Ma et al., 2020a, b), we established a comprehensive single-nucleus transcriptomic atlas of monkey hippocampal aging, which provides extensive resources for the illustration of age-related molecular signatures at the single-cell level. Overall, we have captured a broad spectrum of cell types in the hippocampus, including the neurogenic lineage cells, oligodendrocyte lineage cells, microglia, and other niche cells. While our study failed to capture a cell cluster with the astrocyte signature, it may be attributed to the technological limitation in enriching astrocytes with the current procedures. One possibility is that astrocyte and NSC shared some common marker genes, implying that the NSC population may contain some astrocyte, which was consistent with the previous studies (Artegiani et al., 2017; Dulken et al., 2019). Another technical restriction that snRNA-seq only sequences the pre-mRNA in the nucleus with relatively low sequencing depth may also limit the comprehensiveness and dynamic range of detection, especially for mature neurons with dendrites and axons. In this context, spatial transcriptomic analysis, a newly developed technology (Stahl et al., 2016; Chen et al., 2020), may provide a potential resolution for measuring transcriptional profiles of previously undetectable cells.

Neurogenesis abnormality can lead to compromised neuron regeneration upon injury, along with progressive neurodegeneration and loss of memory in physiological aging (Wyss-Coray, 2016). Since the old hippocampi in the present study were still disease-free, an overall change in their major cell types were not yet presented, nor were the consequent alteration in its structure. Although we did observe reduced $\mathrm{DCX}^{+}$TAPC and increased pro-inflammation $\mathrm{OL}$ in the elderly, not excluding the possibility of changes that may be revealed by future studies to classify hippocampal cells into finer subpopulations. But more importantly, we found significant remodeling in the transcriptomes of various cell types, reflecting dysregulation at multiple steps along the developmental trajectories of the neurogenic lineage cells in the aged NHP hippocampus, i.e., at the early onset of neurogenesis and at the later synaptic transmission stage. Especially, we found age-associated downregulation of NFI family members during the initiation of TAPC differentiation, which are import transcription factors in multiple organ development (Harris et al., 2015; Zenker et al., 2019). In line with our observations, recent studies demonstrated that knockout mice of either Nfia, Nfib, or Nfix displayed shared brain defects, including megalencephaly, dysgenesis of the corpus callosum, and a severely malformed hippocampus with reduced DG volume (Zenker et al., 2019). It was further supported by human phenotypes caused by the haploinsufficiency of NFIA, NFIB, and NFIX (Zenker et al., 2019), suggesting a common but not redundant role of these $\mathrm{NFI}$ members in brain development. Together with our findings, the function of these NFI members now extends to the protection of cognition during aging, providing potential targets to intervene disease progression in the nervous system.

On the other hand, neuroinflammation has also been documented for a crucial role in the pathogenesis of chronic neurodegenerative diseases, such as the progressive loss of memory and development of AD (Ransohoff, 2016; Leng and Edison, 2021). Notably, microglial cells are the primary players in neuroinflammation among the innate immune cells (Ransohoff, 2016). In line with previous observations, our single-nucleus transcriptomic atlas unraveled the upregulation of several pro-inflammatory genes, such as $C D 74$, which can mediate the MIF signaling and promote the inflammatory response (Bryan et al., 2008), as well as the canonical proinflammatory factors TNF- $\alpha$ in the aged microglia. Of note, TNF- $\alpha$ has been reported to promote necroptosis mediated by receptor-interacting protein kinase 1 (RIPK1), RIPK3, and MLKL (Ofengeim and Yuan, 2013), which can robustly elicit the cascade of the inflammation responses, further magnifying the deleterious pro-inflammatory microenvironment that mediates the pathogenesis of various neurodegenerative diseases (Yuan et al., 2019). Interestingly, a recent study has suggested that microglia activated by $A \beta$ deposition could produce neurotoxic cytokines and chemokines, including TNF, IL-6, IL-1 $\beta$ and CCL2, which subsequently cause neuronal dysfunction and death (Leng and Edison, 2021). Consistently, we observed elevated cytosolic $A \beta$ deposition and accumulation of aggregates in the aged hippocampus, further supporting a potential connection between pathological protein aggregates and neuroinflammation in primate hippocampal aging. However, whether and how microglia-mediated age-related neuroinflammation suppress TAPC division and synaptic plasticity remain to be investigated in the future.

To our knowledge, we provided the first evidence that the NHP hippocampus underwent age-dependent genomic and epigenomic alterations, characterized by increased DNA damage, aggravated cytosolic dsDNA release from the nucleus, decondensed heterochromatin, and consequent derepression of heterochromatin-enriched LINE1 retrotransposon (LINE1). Emerging evidence indicates that destability of heterochromatin is a major driver for accelerated stem cell aging, and that consolidation of heterochromatin can rejuvenate aged stem cells (Zhang et al., 2015, 2020b; Deng et al., 2019; Bi et al., 2020; Hu et al., 2020; Diao et al., 2021; Liang et al., 2021). We hypothesized that 
the age-associated perturbation of the nuclear envelope may result in the leakage of the dsDNA and transcripts of derepressed repetitive sequences (Dou et al., 2015; Zhang et al., 2015, 2020b; Buchwalter et al., 2019; De Cecco et al., 2019; Deng et al., 2019; Geng et al., 2019; Simon et al., 2019; Bi et al., 2020; Hu et al., 2020; Diao et al., 2021; Liang et al., 2021; Liu et al., 2021), thus elicit consequent inflammation responses as observed in the aged NHP hippocampus. Consistently, it has been reported that nuclear envelope impairment is tightly associated with aging and neurodegenerative diseases, such as AD and PD (Liu et al., 2012; Frost, 2016; Bedrosian et al., 2021; Bin Imtiaz et al., 2021). When dsDNA accumulates in the cytoplasm, it usually triggers the cGAS-STING signaling pathway and subsequently activates the innate immune responses (Volkman and Stetson, 2014; Bi et al., 2020; Zhang et al., 2020b), which is frequently observed in senescent stem cells and a variety of age-related disorders as age-associated inflammation (De Cecco et al., 2019; Geng et al., 2019; Simon et al., 2019; Bi et al., 2020). Our findings propose a yet-to-be-explored link between (epi)genomic instability and elevated neuroinflammation in primate hippocampal aging.

All in all, by constructing the single-cell resolution transcriptomic atlas of primate hippocampal aging, we aimed to provide a deeper understanding of physiological aging of the primate hippocampus and its pathological relevance. We identified molecular features corresponding to (epi)genomic instability and accumulated damages, elevated neuroinflammation, compromised functions in specific cell types in the old hippocampus, which contribute collectively to the impaired ability for neuronal regeneration, thus enabling the identification of potential diagnostic biomarkers and therapeutic targets for neurodegenerative diseases associated with hippocampal aging.

\section{MATERIALS AND METHODS}

Ethical statement

This study was conducted following the guidelines for the Ethical Treatment of Non-Human Primates and was approved by the Institutional Animal Care and Use Committee of the Institute of Zoology (Chinese Academy of Sciences). All animal experiment procedures were performed by certified veterinarians, complying with laws governing animal research.

\section{Hippocampus tissue collection}

Brain tissues from eight young (4-6 years old) and eight aged (1821 years old) cynomolgus monkeys (Macaca fascicularis) were harvested as previously described (Li et al., 2020; Wang et al., 2020a, b; Zhang et al., 2020c). The monkeys were the same ones sacrificed in the aforementioned studies (Li et al., 2020; Wang et al., 2020a; Zhang et al., 2020c). After the perfusion was completed and the tissue turned white, right hemisphere of each brain was fixed in
$4 \%$ paraformaldehyde for histological analyses. Left hemisphere of each brain was separated according to brain regions and was frozen in liquid nitrogen. The hippocampus is located on the medial temporal lobe. We carefully picked out the hippocampus on ice, divided them into pieces in order, placed them in a cryotube, and stored them in liquid nitrogen (During the collection of frozen tissue, the hippocampus from a young female individual was excluded due to limited technical experience at the beginning of the experiment).

Nissl staining

Nissl staining was performed as previously described (Boldrini et al., 2018). After the hippocampus tissues fixed in $4 \%$ PFA are dehydrated in a series of graded alcohols $(70 \%-100 \%)$, the tissues were embedded in paraffin and sliced to a thickness of $5 \mu \mathrm{m}$ using a rotary microtome. The sections were placed on a microscope slide, dried at $56{ }^{\circ} \mathrm{C}$ for $2 \mathrm{~h}$, and stored at room temperature (RT). For Nissl staining, sections were deparaffinized and rehydrated using xylene and graded alcohols $(100 \%-70 \%)$, and then rinsed in distilled water. Tissue sections were incubated with Nissl Staining Solution (Beyotime, China) at RT for $10 \mathrm{~min}$, and washed with distilled water and $70 \%$ ethanol twice. Sections were quickly dehydrated in $95 \%$ ethanol and $100 \%$ ethanol, cleared in xylene, and mounted with a resinous mounting medium.

\section{SA- $\beta$-Gal staining}

SA- $\beta$-Gal staining was performed using a previously published protocol (Debacq-Chainiaux et al., 2009; Chow et al., 2019; Ma et al., 2020a, 2020b). In brief, the hippocampal tissue stored in the cryotube was fixed and dehydrated in a cold mixture of $75 \%$ ethanol and $4 \%$ paraformaldehyde at $4{ }^{\circ} \mathrm{C}$ shaking for $3 \mathrm{~h}$, then in $4 \%$ paraformaldehyde at $4{ }^{\circ} \mathrm{C}$ shaking overnight, followed by shaking in $5 \%$ sucrose at $4{ }^{\circ} \mathrm{C}$ for $3 \mathrm{~h}$ and then in $30 \%$ sucrose at $4{ }^{\circ} \mathrm{C}$ shaking overnight or until the tissue shrinked completely. OCT-embedded, dehydrated hippocampus tissues were cryosectioned at a thickness of $10 \mu \mathrm{m}$ with a Leica CM3050S cryomicrotome, collected on Superfrost Plus microslides (VWR) and stored at $-80{ }^{\circ} \mathrm{C}$ until use. For SA- $\beta-$ Gal staining, sections were thawed at RT and rinsed in PBS, fixed in $2 \%$ formaldehyde and $0.2 \%$ glutaraldehyde at RT for 5 min and stained with freshly prepared staining solution at $37^{\circ} \mathrm{C}(\mathrm{X}$ gal was purchased from Amresco; all the other reagents were from Sigma-Aldrich). Images were taken with PerkinEImer Vectra Polaris, and the SA- $\beta$-Gal-positive areas were quantified using ImageJ.

\section{Aggresome staining}

Aggresome staining was performed as previously described ( $\mathrm{Li}$ et al., 2020). The paraffin section was deparaffinized and rehydrated using xylene and graded ethanol. Sections were permeabilized with $0.3 \%$ Triton X-100 in PBS for 7 min, incubated with the Aggresome dye (Proteostat ${ }^{8}$ Protein aggregation assay, Enzo, ENZ-51023KP002, 1:3,000 dilution in PBS) for 3 min and then destained in 1\% acetic acid for $30 \mathrm{~min}$. Next, sections were washed with PBS for 3 times (10 min each) at RT, and counterstained with Hoechst 33342 
(Thermo Fisher Scientific). Zeiss 900 confocal system was used for aggresome staining microscopy.

\section{Immunofluorescence staining}

Immunofluorescence staining was performed as described ( $\mathrm{Li}$ et al., 2020; Wang et al., 2020a, b; Zhong et al., 2020). Briefly, paraffinembedded sections were deparaffinized in xylene and rehydrated through gradient ethanol (100\%-70\%). After rinsing in distilled water, sections were microwaved in $10 \mathrm{mmol} / \mathrm{L}$ sodium citrate buffer $(\mathrm{pH}$ 6.0) 5 times for 3 min each. Upon cooling down to RT, sections were rinsed three times in PBS, permeabilized with $0.4 \%$ Triton X-100 in PBS for $2 \mathrm{~h}$ and rinsed again in PBS three times. Sections were then incubated with blocking buffer ( $10 \%$ donkey serum in PBS) at RT for $1 \mathrm{~h}$, followed by incubation with primary antibodies overnight at $4{ }^{\circ} \mathrm{C}$ and fluorescence-labeled secondary antibodies at RT for $1 \mathrm{~h}$. Nuclei were counterstained with Hoechst 33342 (Thermo Fisher Scientific) before the sections were mounted in VECTERSHIELD ${ }^{\circledR}$ anti-fading mounting medium (Vector Laboratories, h-1000). Zeiss 900 confocal system was used for immunofluorescence microscopy. The antibodies used for immunofluorescence staining are as follows: anti- $\beta$ Amyloid (4G8) (Biolegend, 800701, 1:200), anti-H3K9me3 (Abcam, ab8898, 1:500), anti-LINE1 ORF2p (Abcam, ab106004, 1:200), antidsDNA (Santa Cruz Biotechnology, sc-58749, 1:500). Secondary antibodies used were the following: donkey anti rabbit-AF488 (Thermo Fisher (1:500)), donkey anti mouse-AF488 (Thermo Fisher (1:500)), donkey anti chicken-AF488 (Thermo Fisher (1:500)).

\section{Immunohistochemistry staining}

Immunohistochemistry staining was performed as previously described ( $\mathrm{Li}$ et al., 2020; Ma et al., 2020b). Briefly, the paraffinembedded sections were deparaffinized and rehydrated using xylene and graded ethanol. Antigen retrieval was performed by steaming in citrate buffer 5 times for 3 min each. After being cooled down to RT, sections were rinsed three times in PBS, permeabilized with $0.4 \%$ Triton $\mathrm{X}-100$ in PBS for $2 \mathrm{~h}$ and rinsed again in PBS three times. And then sections were incubated with $3 \% \mathrm{H}_{2} \mathrm{O}_{2}$ for $10 \mathrm{~min}$ to inactivate endogenous peroxidase. Sections were then blocked with $10 \%$ donkey serum in PBS for $1 \mathrm{~h}$ and incubated with primary antibodies at $4{ }^{\circ} \mathrm{C}$ overnight. The next day, sections were incubated with HRP-conjugated secondary antibodies at RT for $1 \mathrm{~h}$. Sections were performed using the DAB Staining Kit (ZSGB-BIO, ZLI$9018)$ according to the manufacturer's instructions. Finally, sections were dehydrated in a series of graded alcohols $(50 \%, 70 \%, 80 \%$, $90 \%, 100 \%$, and $100 \%$ ) and xylene before being mounted in the neutral resinous mounting medium. The antibodies used for immunohistochemistry staining are as follows: anti-DCX (Abcam, ab18723, 1:200), anti- $\beta-A m y l o i d ~(1-40)$ (Cell Signaling, 12990s, 1:400), anti-yH2A.X (Millipore, 05636, 1:100), anti-HP1Y (Cell Signaling, 2616s, 1:400), anti-TNF- $\alpha$ (Santa Cruz Biotechnology, sc52746, 1:200). Images were taken with PerkinEImer Vectra Polaris.

\section{Nuclei isolation and snRNA-seq on the 10x Genomics platform}

The protocol for the isolation of nuclei from frozen brain tissues was adapted from Krishnaswami et al. (2016) with minor modifications (Ma et al., 2020a, b). All procedures were carried out on ice or at $4^{\circ}$
C. Nuclei isolation media 1 and 2 (NIM1 and NIM2) and homogenization buffer were pre-chilled on ice. NIM1 contained $250 \mathrm{mmol} / \mathrm{L}$ sucrose, $25 \mathrm{mmol} / \mathrm{L} \mathrm{KCl}, 5 \mathrm{mmol} / \mathrm{L} \mathrm{MgCl}$, $10 \mathrm{mmol} / \mathrm{L}$ Tris buffer $(\mathrm{pH}$ 8.0) in nuclease-free water. NIM2 was the same as NIM1 except for the addition of $1 \mu \mathrm{mol} / \mathrm{L}$ DTT and $1 \times$ protease inhibitor. Homogenization buffer combined the following reagents, including NIM2, 0.4 $\mathrm{U} / \mu \mathrm{L}$ RNaseln, $0.2 \mathrm{U} / \mu \mathrm{L}$ Superasin, $0.1 \%$ Triton $\mathrm{X}-100,1 \mu \mathrm{mol} / \mathrm{L}$ propidium iodide (PI), and $10 \mathrm{ng} / \mathrm{mL}$ Hoechst 33342. The Dounce homogenizer and pestles were pre-chilled on ice. Frozen brain tissues were homogenized with strokes of the pestle in $1 \mathrm{~mL}$ prechilled homogenization buffer. Next, put the tubes into dounce homogenizer, and grind for $60 \mathrm{hz}, 60 \mathrm{~s}, 3$ times. Then the homogenized tissue was filtered through a $40-\mu \mathrm{m}$ cell strainer (BD Falcon), and $500 \mu \mathrm{L}$ supernatant was collected. The nuclei were collected by centrifugation at $1000 \times g$ for $8 \mathrm{~min}$ at $4{ }^{\circ} \mathrm{C}$. Samples were filtered through a $40-\mu \mathrm{m}$ cell strainer, centrifuged at $1000 \times \mathrm{g}$ for $8 \mathrm{~min}$ at $4{ }^{\circ}$ $\mathrm{C}$, and resuspended in PBS supplemented with $0.3 \% \mathrm{BSA}, 0.4 \mathrm{U} / \mu \mathrm{L}$ RNaseln and $0.2 \mathrm{U} / \mu \mathrm{L}$ Superasin. Nuclei were sorted by staining with both Hoechst 33342 and PI using fluorescence-activated cell sorting (FACS) (BD Influx) and counted with a dual-fluorescence cell counter (Luna-FLTM, Logos Biosystems). Mononuclear capture was conducted with a 10x Genomics single-cell 3' system. Approximately 8,000 nuclei were captured for each sample following the standard $10 x$ capture and library preparation protocol (10x Genomics) and then sequenced on a NovaSeq 6000 sequencing platform (Illumina, 20012866).

Processing and quality control of snRNA-seq data

Raw sequencing reads were aligned to the pre-mRNA reference (Ensemble, Macaca_fascicularis_5.0) and counted using Cell Ranger (version 3.1.0) with the default parameters. SoupX (version 1.4.8) was applied to every sample to remove ambient RNA bias (Young and Behjati, 2020). Seurat (version 3.1.3) object of each sample was constructed from decontaminated matrix and cells with genes fewer than 200 or mitochondrial ratio more than $2.5 \%$ were discarded (Butler et al., 2018). Doublet detection was performed with DoubletFinder (version 2.0.2) (McGinnis et al., 2019). After sample integration and clustering as mentioned below, clusters lacking specific marker genes and with relatively low gene content and high mitochondrial ratio were also discarded.

Integration, clustering, and identification of cell types

Dataset of each sample was normalized using the "SCTransform" function of Seurat. Features and anchors for downstream integration were selected with corresponding pipeline according to the SCTransform method, ensuring that calculation was based on all necessary Pearson residuals. After data integration and scaling, principal component analysis was applied with the "RunPCA" function and appropriate principal components were selected for the following analysis. Dimensionality was reduced with the "RunUMAP" function. "FindNeighbors" and "FindClusters" functions were applied to perform clustering. Marker genes for each cluster were identified by the "FindAllMarkers" function with the cutoff of adjusted $P$-values $<0.05$ and $|\log \mathrm{FC}|>1$ (Table S1). Canonical marker genes were used to identify cell types. 
Transcriptional noise analysis

Transcriptional noise was estimated as previously described (Angelidis et al., 2019). In brief, equal number of cells of each cell type were used between the young and old groups. All genes were ordered by their expression levels, and those with the top $10 \%$ and bottom $10 \%$ expression levels were excluded. Transcriptional noise at the cell level was then calculated as the euclidean distances between cells with the mean value of the corresponding cell type. For transcriptional noise at the sample level, the euclidean distances between young and old samples were averaged for each cell type.

Age-related differential gene expression analysis

Differentially expressed genes (DEGs) were identified with the "FindMarkers" function of Seurat in every cell type between young and old samples using the Wilcoxon test with the threshold of adjusted $P$-values $<0.05$ and $|\log F C|>0.25$ (Table S2).

\section{Transcriptional regulatory network analysis}

Transcriptional regulatory network analysis was conducted using SCENIC workflow (version 1.1.2.2) with default parameters based on hg19 database from RcisTarget (version 1.6.0) (Aibar et al., 2017). For cell-type-specific transcription regulatory network, only marker genes were used and calculation was performed in each cell type. And cell types with cell more than 20,000 (microglia and OL) were subsampled to 20,000 cells. For age-related transcriptional regulatory network, only age-related DEGs were used as input for transcriptional regulator inferring and all selected cell types were calculated together. Obtained transcription regulatory network was visualized by Cytoscape (version 3.8.2) (Shannon et al., 2003).

\section{GO term analysis}

FunSet (http://funset.uno/) and Metascape were used to perform GO term (http://metascape.org/gp/index.html) (version 3.5) (Zhou et al., 2019). Results were further visualized with the ggplot2 $R$ package (https://ggplot2.tidyverse.org/) (version 3.2.1).

\section{Pseudotime analysis}

Pseudotime analysis was performed with using the Monocle2 R package (Trapnell et al., 2014). Top 200 highly variable genes from the "FindMarkers" function of Seurat package were used as ordering genes with the threshold of adjusted $P$-value $<0.05$ and $|\log F C|>1$. DDRTree dimensionality reduction method was applied to construct the trajectory that was plotted in two-dimensional space. Differentiation-related DEGs were obtain with a cutoff of $q$ value $<1 \times 10^{-4}$.

\section{Cell-cell communication analysis}

Cell-cell communication analysis was performed using the CellPhoneDB (version 1.1.0) (Efremova et al., 2020). Only receptors and ligands expressed in more than $10 \%$ cells of any cell type from either young or old samples were further evaluated. Only those with a $P$ value $<0.01$ were used for the prediction of cell-cell communication between any two cell types.
Gene set score analysis

Gene sets related to aging-related diseases were obtained from database DisGeNET (https://www.disgenet.org/home/). Gene sets of $A D$ and $P D$ were generated by filtering with "diseaseName" ("Alzheimer's disease" and "Parkinson disease", respectively). Gene set of learning disorders consists of genes related to "Learning Disorders", "Learning Disturbance" and "Learning Disabilities", while that of memory disorders includes genes related to "Age-Related Memory Disorders", "Memory Disorders", "Memory impairment", "Memory Disorder, Semantic", "Memory Disorder, Spatial" and "Memory Loss". Other gene sets used in this paper were acquired from GSEA (Subramanian et al., 2005). Genes related to pro-/anticoagulation and SASP were manually curated from previous literatures (Hoppe and Dorner, 2012; Ma et al., 2020a, b; Gu et al., 2021). All gene sets involved in this study are summarized in Table S6. Gene set scores were acquired by analyzing the transcriptome of each input cell against aforementioned gene sets by the Seurat function "AddModuleScore". Changes in the scores between young and old samples were analyzed using ggpubr $R$ package via the Wilcoxon test (https://github.com/kassambara/ggpubr) (version $0.2 .4)$.

\section{Statistical analysis}

All experimental data were statistically analyzed using PRISM software (GraphPad 8 Software). Results were presented as mean \pm SEM. Comparisons were conducted using the two-tailed Student's $t$ test.

\section{DATA AVAILABILITY}

All the sequencing data are deposited in Genome Sequence Archive (GSA) (https://bigd.big.ac.cn/gsa/) with the accession number of CRA004080 and the snRNA-seq atlas can be accessed via an interactive user-friendly webtool at Aging Atlas (https://bigd.big.ac. cn/aging/landscape?project=CM_hippocampus) (Aging Atlas, 2021).

\section{ACKNOWLEDGEMENTS}

We thank Shanshan Che, Liyun Zhao, Xiaoyan Sun, and Yixin Zhang for their help in immunofluorescence staining, Ruotong Ren, Liping Deng and Xiaojuan He for their help in tissue collection, Junying Jia from the Institute of Biophysics, Chinese Academy of Sciences for his help in fluorescence-activated cell sorting (FACS), as well as Shiwen Li from the Institute of Zoology, Chinese Academy of Sciences for her help in image scanning of immunohistochemical staining. We thank Profs. Fuchou Tang, Xiaoqun Wang, Young Shen, Moshi Song, Fudong Shi and Junying Yuan for their helpful suggestions and discussions. We are grateful to Lei Bai, Qun Chu, Xiao Zhuo, Jing Lu, Ying Yang, Ruijun Bai, and Shikun Ma for administrative assistance. This work was supported by the National Key Research and Development Program of China (2020YFA0804000), the Strategic Priority Research Program of the Chinese Academy of Sciences (XDA16010000), the National Key Research and Development Program of China (2019YFA0110100, 2020YFA0112201, 2018 
YFC2000100, 2017YFA0103304, 2017YFA0102802, 2018Y FA0107203, 2020YFA0803401, and 2019YFA0802202), the National Natural Science Foundation of China (Grant Nos. 81921006, 81625009, 91749202, 81861168034, 91949209, 92049304, 81822018, 82071588, 92049116, 31900523, 32000500, 31970597, 82030037, and 81801534), the Program of the Beijing Municipal Science and Technology Commission (Z191100001519005), Beijing Natural Science Foundation (Z190019), the Key Research Program of the Chinese Academy of Sciences (KFZD-SW-221), K. C. Wong Education Foundation (GJTD-2019-06, GJTD-2019-08), the International Partnership Program of Chinese Academy of Sciences (152111KYSB20160004), the Non-profit Central Research Institute Fund of Chinese Academy of Medical Sciences (2020-JKCS-011), the State Key Laboratory of Stem Cell and Reproductive Biology, the State Key Laboratory of Membrane Biology, the Milky Way Research Foundation (MWRF), and the Moxie Foundation (for J.C.I.B.).

\section{OPEN ACCESS}

This article is licensed under a Creative Commons Attribution 4.0 International License, which permits use, sharing, adaptation, distribution and reproduction in any medium or format, as long as you give appropriate credit to the original author(s) and the source, provide a link to the Creative Commons licence, and indicate if changes were made. The images or other third party material in this article are included in the article's Creative Commons licence, unless indicated otherwise in a credit line to the material. If material is not included in the article's Creative Commons licence and your intended use is not permitted by statutory regulation or exceeds the permitted use, you will need to obtain permission directly from the copyright holder. To view a copy of this licence, visit http:// creativecommons.org/licenses/by/4.0/.

\section{REFERENCES}

Aging Atlas C (2021) Aging Atlas: a multi-omics database for aging biology. Nucleic Acids Res 49:D825-D830

Aibar S, González-Blas CB, Moerman T, Huynh-Thu VA, Imrichova $\mathrm{H}$, Hulselmans G, Rambow F, Marine J-C, Geurts P, Aerts J et al (2017) SCENIC: single-cell regulatory network inference and clustering. Nat Methods 14:1083-1086

Aimone JB, Li Y, Lee SW, Clemenson GD, Deng W, Gage FH (2014)

Regulation and function of adult neurogenesis: from genes to cognition. Physiol Rev 94:991-1026

Angelidis I, Simon LM, Fernandez IE, Strunz M, Mayr CH, Greiffo FR, Tsitsiridis G, Ansari M, Graf E, Strom TM et al (2019) An atlas of the aging lung mapped by single cell transcriptomics and deep tissue proteomics. Nat Commun 10:963

Artegiani B, Lyubimova A, Muraro M, van Es JH, van Oudenaarden A, Clevers $H$ (2017) A single-cell RNA sequencing study reveals cellular and molecular dynamics of the hippocampal neurogenic niche. Cell Rep 21:3271-3284

Baird GS, Nelson SK, Keeney TR, Stewart A, Williams S, Kraemer S, Peskind ER, Montine TJ (2012) Age-dependent changes in the cerebrospinal fluid proteome by slow off-rate modified aptamer array. Am J Pathol 180:446-456
Baker DJ, Petersen RC (2018) Cellular senescence in brain aging and neurodegenerative diseases: evidence and perspectives. J Clin Investig 128:1208-1216

Bedrosian TA, Houtman J, Eguiguren JS, Ghassemzadeh S, Rund N, Novaresi NM, Hu L, Parylak SL, Denli AM, Randolph-Moore L et al (2021) Lamin B1 decline underlies age-related loss of adult hippocampal neurogenesis. EMBO J 40:e105819

Bengoa-Vergniory N, Kypta RM (2015) Canonical and noncanonical Wnt signaling in neural stem/progenitor cells. Cell Mol Life Sci 72:4157-4172

Bi S, Liu Z, Wu Z, Wang Z, Liu X, Wang S, Ren J, Yao Y, Zhang W, Song $M$ et al (2020) SIRT7 antagonizes human stem cell aging as a heterochromatin stabilizer. Protein Cell 11:483-504

Bin Imtiaz MK, Jaeger BN, Bottes S, Machado RAC, Vidmar M, Moore DL, Jessberger S (2021) Declining lamin B1 expression mediates age-dependent decreases of hippocampal stem cell activity. Cell Stem Cell. https://doi.org/10.1016/j.stem.2021.01. 015

Boldrini M, Fulmore CA, Tartt AN, Simeon LR, Pavlova I, Poposka V, Rosoklija GB, Stankov A, Arango V, Dwork AJ et al (2018) Human hippocampal neurogenesis persists throughout aging. Cell Stem Cell 22(589-599):e585

Brunk UT, Terman A (2002) The mitochondrial-lysosomal axis theory of aging. Eur J Biochem 269:1996-2002

Bryan KJ, Zhu X, Harris PL, Perry G, Castellani RJ, Smith MA, Casadesus G (2008) Expression of CD74 is increased in neurofibrillary tangles in Alzheimer's disease. Mol Neurodegener $3: 13$

Buchwalter A, Kaneshiro JM, Hetzer MW (2019) Coaching from the sidelines: the nuclear periphery in genome regulation. Nat Rev Genet 20:39-50

Buckig A, Tikkanen R, Herzog V, Schmitz A (2002) Cytosolic and nuclear aggregation of the amyloid beta-peptide following its expression in the endoplasmic reticulum. Histochem Cell Biol 118:353-360

Butler A, Hoffman P, Smibert P, Papalexi E, Satija R (2018) Integrating single-cell transcriptomic data across different conditions, technologies, and species. Nat Biotechnol 36:411-420

Chen Y, Niu Y, Ji W (2012) Transgenic nonhuman primate models for human diseases: approaches and contributing factors. J Genet Genom 39:247-251

Chen Y, Niu Y, Ji W (2016) Genome editing in nonhuman primates: approach to generating human disease models. J Intern Med 280:246-251

Chen Y, Yu J, Niu Y, Qin D, Liu H, Li G, Hu Y, Wang J, Lu Y, Kang Y et al (2017) Modeling Rett syndrome using TALEN-edited MECP2 mutant cynomolgus monkeys. Cell 169(945-955):

Chen WT, Lu A, Craessaerts K, Pavie B, Sala Frigerio C, Corthout N, Qian X, Lalakova J, Kuhnemund M, Voytyuk I et al (2020) Spatial transcriptomics and in situ sequencing to study Alzheimer's disease. Cell 182(976-991):e919

Chow HM, Shi M, Cheng A, Gao Y, Chen G, Song X, So RWL, Zhang J, Herrup K (2019) Age-related hyperinsulinemia leads to insulin resistance in neurons and cell-cycle-induced senescence. Nat Neurosci 22:1806-1819

Colman RJ (2018) Non-human primates as a model for aging. Biochim Biophys Acta Mol Basis Dis 1864:2733-2741 
Costa-Mattioli M, Walter P (2020) The integrated stress response: from mechanism to disease. Science 368:eaat5314

De Cecco M, Ito T, Petrashen AP, Elias AE, Skvir NJ, Criscione SW, Caligiana A, Brocculi G, Adney EM, Boeke JD et al (2019) L1 drives IFN in senescent cells and promotes age-associated inflammation. Nature 566:73-78

Debacq-Chainiaux F, Erusalimsky JD, Campisi J, Toussaint O (2009) Protocols to detect senescence-associated beta-galactosidase (SA-ßgal) activity, a biomarker of senescent cells in culture and in vivo. Nat Protoc 4:1798-1806

Deng L, Ren R, Liu Z, Song M, Li J, Wu Z, Ren X, Fu L, Li W, Zhang $W$ et al (2019) Stabilizing heterochromatin by DGCR8 alleviates senescence and osteoarthritis. Nat Commun 10:3329

Diao Z, Ji Q, Wu Z, Zhang W, Cai Y, Wang Z, Hu J, Liu Z, Wang Q, Bi $S$ et al (2021) SIRT3 consolidates heterochromatin and counteracts senescence. Nucleic Acids Res 49:4203-4219

Dou Z, Xu C, Donahue G, Shimi T, Pan JA, Zhu J, Ivanov A, Capell BC, Drake AM, Shah PP et al (2015) Autophagy mediates degradation of nuclear lamina. Nature 527:105-109

Dulken BW, Buckley MT, Navarro Negredo P, Saligrama N, Cayrol R, Leeman DS, George BM, Boutet SC, Hebestreit K, Pluvinage JV et al (2019) Single-cell analysis reveals T cell infiltration in old neurogenic niches. Nature 571:205-210

Efremova M, Vento-Tormo M, Teichmann SA, Vento-Tormo R (2020) Cell PhoneDB: inferring cell-cell communication from combined expression of multi-subunit ligand-receptor complexes. Nat Protoc 15:1484-1506

Encinas JM, Michurina TV, Peunova N, Park JH, Tordo J, Peterson DA, Fishell G, Koulakov A, Enikolopov G (2011) Division-coupled astrocytic differentiation and age-related depletion of neural stem cells in the adult hippocampus. Cell Stem Cell 8:566-579

Fan X, Wheatley EG, Villeda SA (2017) Mechanisms of hippocampal aging and the potential for rejuvenation. Annu Rev Neurosci 40:251-272

Frost B (2016) Alzheimer's disease: an acquired neurodegenerative laminopathy. Nucleus 7:275-283

Geng L, Liu Z, Wang S, Sun S, Ma S, Liu X, Chan P, Sun L, Song M, Zhang $W$ et al (2019) Low-dose quercetin positively regulates mouse healthspan. Protein Cell 10:770-775

Geutskens SB, Hordijk PL, van Hennik PB (2010) The chemorepellent Slit3 promotes monocyte migration. J Immunol 185:76917698

Giacobini E, Gold G (2013) Alzheimer disease therapy-moving from amyloid-beta to tau. Nat Rev Neurol 9:677-686

Gu SX, Tyagi T, Jain K, Gu VW, Lee SH, Hwa JM, Kwan JM, Krause DS, Lee Al, Halene $S$ et al (2021) Thrombocytopathy and endotheliopathy: crucial contributors to COVID-19 thromboinflammation. Nat Rev Cardiol 18:194-209

Gust J, Hay KA, Hanafi LA, Li D, Myerson D, Gonzalez-Cuyar LF, Yeung C, Liles WC, Wurfel M, Lopez JA et al (2017) Endothelial activation and blood-brain barrier disruption in neurotoxicity after adoptive immunotherapy with CD19 CAR-T cells. Cancer Discov 7:1404-1419

Habib N, Li Y, Heidenreich M, Swiech L, Avraham-Davidi I, Trombetta JJ, Hession C, Zhang F, Regev A (2016) Div-Seq: single-nucleus RNA-Seq reveals dynamics of rare adult newborn neurons. Science 353:925-928
Harris L, Genovesi LA, Gronostajski RM, Wainwright BJ, Piper M (2015) Nuclear factor one transcription factors: divergent functions in developmental versus adult stem cell populations. Dev Dyn 244:227-238

He G, Luo W, Li P, Remmers C, Netzer WJ, Hendrick J, Bettayeb K, Flajolet M, Gorelick F, Wennogle LP et al (2010) Gammasecretase activating protein is a therapeutic target for Alzheimer's disease. Nature 467:95-98

He X, Memczak S, Qu J, Belmonte JCI, Liu GH (2020) Single-cell omics in ageing: a young and growing field. Nat Metab 2:293-302

Head D, Snyder AZ, Girton LE, Morris JC, Buckner RL (2005) Frontal-hippocampal double dissociation between normal aging and Alzheimer's disease. Cereb Cortex 15:732-739

Herculano-Houzel S (2009) The human brain in numbers: a linearly scaled-up primate brain. Front Hum Neurosci 3:31

Hoppe B, Dorner T (2012) Coagulation and the fibrin network in rheumatic disease: a role beyond haemostasis. Nat Rev Rheumatol 8:738-746

Hou Y, Dan X, Babbar M, Wei Y, Hasselbalch SG, Croteau DL, Bohr VA (2019) Ageing as a risk factor for neurodegenerative disease. Nat Rev Neurol 15:565-581

Hu H, Ji Q, Song M, Ren J, Liu Z, Wang Z, Liu X, Yan K, Hu J, Jing Y et al (2020) ZKSCAN3 counteracts cellular senescence by stabilizing heterochromatin. Nucleic Acids Res 48:6001-6018

Hwang IK, Park JH, Lee TK, Kim DW, Yoo KY, Ahn JH, Kim YH, Cho $\mathrm{JH}$, Kim YM, Won MH et al (2017) CD74-immunoreactive activated M1 microglia are shown late in the gerbil hippocampal CA1 region following transient cerebral ischemia. Mol Med Rep 15:4148-4154

Ibrayeva A, Bay M, Pu E, Jorg DJ, Peng L, Jun H, Zhang N, Aaron $D$, Lin C, Resler G et al (2021) Early stem cell aging in the mature brain. Cell Stem Cell. https://doi.org/10.1016/j.stem.2021.03.018

Jin WN, Shi K, He W, Sun JH, Van Kaer L, Shi FD, Liu Q (2021) Neuroblast senescence in the aged brain augments natural killer cell cytotoxicity leading to impaired neurogenesis and cognition. Nat Neurosci 24:61-73

Kempermann G, Song H, Gage FH (2015) Neurogenesis in the Adult Hippocampus. Cold Spring Harb Perspect Biol 7:a018812

Keren-Shaul H, Spinrad A, Weiner A, Matcovitch-Natan O, DvirSzternfeld R, Ulland TK, David E, Baruch K, Lara-Astaiso D, Toth $B$ et al (2017) A unique microglia type associated with restricting development of Alzheimer's disease. Cell 169:1276-1290

Krishnaswami SR, Grindberg RV, Novotny M, Venepally P, Lacar B, Bhutani K, Linker SB, Pham S, Erwin JA, Miller JA et al (2016) Using single nuclei for RNA-seq to capture the transcriptome of postmortem neurons. Nat Protoc 11:499-524

Kruithof EK, Dunoyer-Geindre S (2014) Human tissue-type plasminogen activator. Thromb Haemost 112:243-254

Kuhn HG, Toda T, Gage FH (2018) Adult hippocampal neurogenesis: a coming-of-age story. J Neurosci 38:10401-10410

Leng F, Edison P (2021) Neuroinflammation and microglial activation in Alzheimer disease: where do we go from here? Nat Rev Neurol 17:157-172

Leuner B, Kozorovitskiy Y, Gross CG, Gould E (2007) Diminished adult neurogenesis in the marmoset brain precedes old age. Proc Natl Acad Sci USA 104:17169-17173 
Leyns CEG, Ulrich JD, Finn MB, Stewart FR, Koscal LJ, Remolina Serrano J, Robinson GO, Anderson E, Colonna M, Holtzman DM (2017) TREM2 deficiency attenuates neuroinflammation and protects against neurodegeneration in a mouse model of tauopathy. Proc Natl Acad Sci USA 114:11524-11529

Li R, Lindholm K, Yang LB, Yue X, Citron M, Yan R, Beach T, Sue L, Sabbagh M, Cai H et al (2004) Amyloid beta peptide load is correlated with increased beta-secretase activity in sporadic Alzheimer's disease patients. Proc Natl Acad Sci USA 101:36323637

Li D, Takeda N, Jain R, Manderfield LJ, Liu F, Li L, Anderson SA, Epstein JA (2015) Hopx distinguishes hippocampal from lateral ventricle neural stem cells. Stem Cell Res 15:522-529

Li J, Zheng Y, Yan P, Song M, Wang S, Sun L, Liu Z, Ma S, Belmonte $\mathrm{JCl}$, Chan $\mathrm{P}$ et al (2020) A single-cell transcriptomic atlas of primate pancreatic islet aging. Natl Sci Rev 8(2):127

Liang C, Liu Z, Song M, Li W, Wu Z, Wang Z, Wang Q, Wang S, Yan $K$, Sun L et al (2021) Stabilization of heterochromatin by CLOCK promotes stem cell rejuvenation and cartilage regeneration. Cell Res 31:187-205

Linnartz-Gerlach B, Bodea LG, Klaus C, Ginolhac A, Halder R, Sinkkonen L, Walter J, Colonna M, Neumann H (2019) TREM2 triggers microglial density and age-related neuronal loss. Glia 67:539-550

Liu GH, Qu J, Suzuki K, Nivet E, Li M, Montserrat N, Yi F, Xu X, Ruiz $S$, Zhang $W$ et al (2012) Progressive degeneration of human neural stem cells caused by pathogenic LRRK2. Nature 491:603-607

Liu X, Liu Z, Sun L, Ren J, Wu Z, Jiang X, Ji Q, Wang Q, Fan Y, Cai $Y$ et al (2021) Resurrection of human endogenous retroviruses during aging reinforces senescence. bioRxiv. https://doi.org/10. 1101/2021.02.22.432260v1.abstract

Lubbe SJ, Bustos B, Hu J, Krainc D, Joseph T, Hehir J, Tan M, Zhang W, Escott-Price V, Williams NM et al (2021) Assessing the relationship between monoallelic PRKN mutations and Parkinson's risk. Human Mol Genet 30:78-86

Ma S, Sun S, Geng L, Song M, Wang W, Ye Y, Ji Q, Zou Z, Wang S, $\mathrm{He} X$ et al (2020a) Caloric restriction reprograms the single-cell transcriptional landscape of rattus norvegicus aging. Cell 180 (984-1001):e1022

Ma S, Sun S, Li J, Fan Y, Qu J, Sun L, Wang S, Zhang Y, Yang S, Liu $Z$ et al (2020b) Single-cell transcriptomic atlas of primate cardiopulmonary aging. Cell Res 31(4):415-432

Malykhin NV, Bouchard TP, Camicioli R, Coupland NJ (2008) Aging hippocampus and amygdala. NeuroReport 19:543-547

Marcos-Contreras OA, Martinez de Lizarrondo S, Bardou I, Orset C, Pruvost M, Anfray A, Frigout Y, Hommet Y, Lebouvier L, Montaner $J$ et al (2016) Hyperfibrinolysis increases blood-brain barrier permeability by a plasmin- and bradykinin-dependent mechanism. Blood 128:2423-2434

Marques S, Zeisel A, Codeluppi S, van Bruggen D, Mendanha Falcao A, Xiao L, Li H, Haring M, Hochgerner H, Romanov RA et al (2016) Oligodendrocyte heterogeneity in the mouse juvenile and adult central nervous system. Science 352:1326-1329

Martinelli P, Real FX (2019) Mouse models shed light on the SLIT/ ROBO pathway in pancreatic development and cancer. Trends Cancer 5:145-148
Mauffrey P, Tchitchek N, Barroca V, Bemelmans A-P, Firlej V, Allory Y, Roméo P-H, Magnon C (2019) Progenitors from the central nervous system drive neurogenesis in cancer. Nature 569:672678

McGinnis CS, Murrow LM, Gartner ZJ (2019) DoubletFinder: doublet detection in single-cell RNA sequencing data using artificial nearest neighbors. Cell Syst 8:329-337.e324

Morrison JH, Baxter MG (2012) The ageing cortical synapse: hallmarks and implications for cognitive decline. Nat Rev Neurosci 13:240-250

Nakamura R, Nakamoto C, Obama H, Durward E, Nakamoto M (2012) Structure-function analysis of Nel, a thrombospondin-1like glycoprotein involved in neural development and functions. J Biol Chem 287:3282-3291

Navarro Negredo P, Yeo RW, Brunet A (2020) Aging and rejuvenation of neural stem cells and their niches. Cell Stem Cell 27:202223

Ofengeim D, Yuan J (2013) Regulation of RIP1 kinase signalling at the crossroads of inflammation and cell death. Nat Rev Mol Cell Biol 14:727-736

Ransohoff RM (2016) How neuroinflammation contributes to neurodegeneration. Science 353:777-783

Rivero O, Sich S, Popp S, Schmitt A, Franke B, Lesch KP (2013) Impact of the ADHD-susceptibility gene $\mathrm{CDH} 13$ on development and function of brain networks. Eur Neuropsychopharmacol 23:492-507

Shannon P, Markiel A, Ozier O, Baliga NS, Wang JT, Ramage D, Amin N, Schwikowski B, Ideker T (2003) Cytoscape: a software environment for integrated models of biomolecular interaction networks. Genome Res 13:2498-2504

Shi Z, Geng Y, Liu J, Zhang H, Zhou L, Lin Q, Yu J, Zhang K, Liu J, Gao $X$ et al (2018) Single-cell transcriptomics reveals gene signatures and alterations associated with aging in distinct neural stem/progenitor cell subpopulations. Protein Cell 9:351-364

Simon M, Van Meter M, Ablaeva J, Ke Z, Gonzalez RS, Taguchi T, De Cecco M, Leonova KI, Kogan V, Helfand SL et al (2019) LINE1 derepression in aged wild-type and SIRT6-deficient mice drives inflammation. Cell Metab 29:871-885

Stahl PL, Salmen F, Vickovic S, Lundmark A, Navarro JF, Magnusson J, Giacomello S, Asp M, Westholm JO, Huss M et al (2016) Visualization and analysis of gene expression in tissue sections by spatial transcriptomics. Science 353:78-82

Su H, Na N, Zhang X, Zhao Y (2017) The biological function and significance of CD74 in immune diseases. Inflamm Res 66:209216

Subramanian A, Tamayo P, Mootha VK, Mukherjee S, Ebert BL, Gillette MA, Paulovich A, Pomeroy SL, Golub TR, Lander ES et al (2005) Gene set enrichment analysis: a knowledge-based approach for interpreting genome-wide expression profiles. Proc Natl Acad Sci 102:15545

Sweeney MD, Sagare AP, Zlokovic BV (2018) Blood-brain barrier breakdown in Alzheimer disease and other neurodegenerative disorders. Nat Rev Neurol 14:133-150

Tanaka T, Biancotto A, Moaddel R, Moore AZ, Gonzalez-Freire M, Aon MA, Candia J, Zhang P, Cheung F, Fantoni G et al (2018) Plasma proteomic signature of age in healthy humans. Aging Cell 17: 
Tiensuu H, Haapalainen AM, Karjalainen MK, Pasanen A, Huusko JM, Marttila R, Ojaniemi M, Muglia LJ, Hallman M, Ramet M (2019) Risk of spontaneous preterm birth and fetal growth associates with fetal SLIT2. PLoS Genet 15:e1008107

Tilstra JS, Clauson CL, Niedernhofer LJ, Robbins PD (2011) NFkappaB in aging and disease. Aging Dis 2:449-465

Trapnell C, Cacchiarelli D, Grimsby J, Pokharel P, Li S, Morse M, Lennon NJ, Livak KJ, Mikkelsen TS, Rinn JL (2014) The dynamics and regulators of cell fate decisions are revealed by pseudotemporal ordering of single cells. Nat Biotechnol 32:381386

Ulland TK, Colonna M (2018) TREM2-a key player in microglial biology and Alzheimer disease. Nat Rev Neurol 14:667-675

Vanlandewijck M, He L, Mae MA, Andrae J, Ando K, Del Gaudio F, Nahar K, Lebouvier T, Lavina B, Gouveia L et al (2018) A molecular atlas of cell types and zonation in the brain vasculature. Nature 554:475-480

Végh MJ, Rausell A, Loos M, Heldring CM, Jurkowski W, van Nierop P, Paliukhovich I, Li KW, del Sol A, Smit AB et al (2014) Hippocampal extracellular matrix levels and stochasticity in synaptic protein expression increase with age and are associated with age-dependent cognitive decline. Mol Cell Proteom 13:2975-2985

Volkman HE, Stetson DB (2014) The enemy within: endogenous retroelements and autoimmune disease. Nat Immunol 15:415422

Wang L, Song G, Zhang X, Feng T, Pan J, Chen W, Yang M, Bai X, Pang Y, Yu J et al (2017) PADI2-mediated citrullination promotes prostate cancer progression. Cancer Res 77:5755-5768

Wang S, Zheng Y, Li J, Yu Y, Zhang W, Song M, Liu Z, Min Z, Hu H, Jing $Y$ et al (2020a) Single-cell transcriptomic atlas of primate ovarian aging. Cell 180(585-600):e519

Wang S, Zheng Y, Li Q, He X, Ren R, Zhang W, Song M, Hu H, Liu F, Sun $G$ et al (2020b) Deciphering primate retinal aging at singlecell resolution. Protein Cell. https://doi.org/10.1007/s13238-02000791-x

Wegiel J, Frackowiak J, Mazur-Kolecka B, Schanen NC, Cook EH Jr, Sigman M, Brown WT, Kuchna I, Wegiel J, Nowicki K et al (2012) Abnormal intracellular accumulation and extracellular Abeta deposition in idiopathic and Dup15q11.2-q13 autism spectrum disorders. PLoS One 7:e35414

Woo MS, Ufer F, Rothammer N, Di Liberto G, Binkle L, Haferkamp U, Sonner JK, Engler JB, Hornig S, Bauer S et al (2021) Neuronal metabotropic glutamate receptor 8 protects against neurodegeneration in CNS inflammation. J Exp Med. https://doi.org/10.1084/ jem.20201290

Wyss-Coray T (2016) Ageing, neurodegeneration and brain rejuvenation. Nature 539:180-186
Yang X, Goh A, Chen SH, Qiu A (2013) Evolution of hippocampal shapes across the human lifespan. Hum Brain Mapp 34:30753085

Yang AC, Stevens MY, Chen MB, Lee DP, Stahli D, Gate D, Contrepois K, Chen W, Iram T, Zhang L et al (2020) Physiological blood-brain transport is impaired with age by a shift in transcytosis. Nature 583:425-430

Young MD, Behjati S (2020) SoupX removes ambient RNA contamination from droplet-based single-cell RNA sequencing data. GigaScience 9:giaa151

Yu HC, Tung CH, Huang KY, Huang HB, Lu MC (2020) The essential role of peptidylarginine deiminases 2 for cytokines secretion, apoptosis, and cell adhesion in macrophage. Int $\mathrm{J}$ Mol Sci 21:5720

Yuan J, Amin P, Ofengeim D (2019) Necroptosis and RIPK1mediated neuroinflammation in CNS diseases. Nat Rev Neurosci 20:19-33

Zenker M, Bunt J, Schanze I, Schanze D, Piper M, Priolo M, Gerkes EH, Gronostajski RM, Richards LJ, Vogt J et al (2019) Variants in nuclear factor I genes influence growth and development. Am J Med Genet C Semin Med Genet 181:611-626

Zhang W, Li J, Suzuki K, Qu J, Wang P, Zhou J, Liu X, Ren R, Xu X, Ocampo A et al (2015) Aging stem cells. A Werner syndrome stem cell model unveils heterochromatin alterations as a driver of human aging. Science 348:1160-1163

Zhang W, Wan H, Feng G, Qu J, Wang J, Jing Y, Ren R, Liu Z, Zhang L, Chen $Z$ et al (2018) SIRT6 deficiency results in developmental retardation in cynomolgus monkeys. Nature 560:661-665

Zhang K, Wang Y, Fan T, Zeng C, Sun ZS (2020a) The p21-activated kinases in neural cytoskeletal remodeling and related neurological disorders. Protein Cell. https://doi.org/10.1007/s13238-02000812-9

Zhang W, Qu J, Liu GH, Belmonte JCl (2020b) The ageing epigenome and its rejuvenation. Nat Rev Mol Cell Biol 21:137150

Zhang W, Zhang S, Yan P, Ren J, Song M, Li J, Lei J, Pan H, Wang $S$, Ma X et al (2020c) A single-cell transcriptomic landscape of primate arterial aging. Nat Commun 11:2202

Zhong S, Ding W, Sun L, Lu Y, Dong H, Fan X, Liu Z, Chen R, Zhang $S$, Ma $Q$ et al (2020) Decoding the development of the human hippocampus. Nature 577:531-536

Zhou Y, Zhou B, Pache L, Chang M, Khodabakhshi AH, Tanaseichuk O, Benner C, Chanda SK (2019) Metascape provides a biologistoriented resource for the analysis of systems-level datasets. Nat Commun 10:1523 